\title{
Theory of the Magnetic Catalysis of Chiral Symmetry Breaking in QED
}

\author{
V.P. Gusynin ${ }^{1}$, V.A. Miransky ${ }^{1}$, and I.A. Shovkovy ${ }^{2 *}$ \\ 1 Bogolyubov Institute for Theoretical Physics, 252143, Kiev, Ukraine \\ ${ }^{2}$ Physics Department, University of Cincinnati, Cincinnati, Ohio 45221-0011
}

(July 25, 2018)

\begin{abstract}
The theory of the magnetic catalysis of chiral symmetry breaking in QED is developed. An approximation for the Schwinger-Dyson equations describing reliably this phenomenon is established, i.e., it is shown that there exists a consistent truncation of those equations in this problem. The equations are solved both analytically and numerically, and the dynamical mass of fermions is determined.
\end{abstract}

11.30.Rd, 11.30.Qc, 12.20.-m

Keywords: quantum electrodynamics, chiral symmetry, magnetic field.

\section{INTRODUCTION}

Recently the magnetic catalysis of dynamical chiral symmetry breaking has been established as a universal phenomenon in $2+1$ and $3+1$ dimensions: a constant magnetic field leads to the generation of a fermion dynamical mass even at the weakest attractive interaction between fermions [1 13$]$. The essence of this effect is the dimensional reduction $D \rightarrow D-2$ in the dynamics of fermion pairing in a magnetic field: at weak coupling, this dynamics is dominated by the lowest Landau level (LLL) which is essentially $(D-2)$-dimensional [1 3 ]. The effect may have interesting applications in condensed matter physics [4] and cosmology [1, 5 , 8 .

In particular, this phenomenon was considered in 3+1 dimensional QED [2, 3.5, 0,9-15]. Since the dynamics of the LLL is long-range (infrared), and the QED coupling constant is weak in the infrared region, one may think that the rainbow (ladder) approximation is reliable in this problem. As was shown in Refs. [2, 3.,6], in all the covariant gauges, the dynamical mass of fermions in this approximation is

$$
m_{d y n}=C \sqrt{|e B|} \exp \left[-\frac{\pi}{2}\left(\frac{\pi}{2 \alpha}\right)^{1 / 2}\right]
$$

where $B$ is a magnetic field, the constant $C$ is of order one and $\alpha$ is the renormalized coupling constant related to the scale $\mu^{2} \sim|e B|$.

Are higher order contributions indeed suppressed in this problem? The answer is "no". As was shown in Refs. [3,5], because of the (1+1)-dimensional form of the fermion propagator of the LLL fermions, there are relevant higher order contributions. In particular, considering this problem in the improved rainbow approximation (with the bare vertex in the Schwinger-Dyson equations for both the fermion propagator and the polarization operator), it was shown that, in all the covariant gauges, the fermion mass $m_{d y n}$ is given by Eq. (11) but with $\alpha \rightarrow \alpha / 2$ [3].

As we wrote in the paper [3], "it is a challenge to define the class of all those diagrams in QED in a magnetic field that give a relevant contribution in this problem". In this paper, we will solve the problem. (A brief outline of our results was given in Ref. [16].) We will show that there exists a (non-covariant) gauge in which the Schwinger-Dyson equations written in the improved rainbow approximation are reliable: in other words, in that gauge, there exists a consistent truncation of the Schwinger-Dyson equations for this non-perturbative problem. The expression for $m_{d y n}$ takes the following form,

$$
m_{d y n}=\tilde{C} \sqrt{|e B|} F(\alpha) \exp \left[-\frac{\pi}{\alpha \ln \left(C_{1} / N \alpha\right)}\right]
$$

where $N$ is the number of fermion flavors, $F(\alpha) \simeq(N \alpha)^{1 / 3}, C_{1} \simeq 1.82 \pm 0.06$ and the constant $\tilde{C}$ is of order one.

This expression for $m_{d y n}$ is essentially different from that in the rainbow approximation (1). As we will see, this reflects rather rich and sophisticated dynamics in this problem.

*On leave of absence from Bogolyubov Institute for Theoretical Physics, 252143, Kiev, Ukraine. 
The paper is organized as follows. In Section II the Schwinger-Dyson equations in QED in a magnetic field are discussed. In Section III we define a (non-covariant) gauge in which the improved rainbow approximation for these equations is reliable. In Section IV the loop expansion for the Schwinger-Dyson equations is considered and it is confirmed that the improved rainbow approximation is indeed reliable in the gauge introduced in Section III. In Section $\mathrm{V}$ the Schwinger-Dyson equations in this approximation are solved both analytically and numerically. In Section VI we summarize the main results of the paper. The details of our analysis and some useful formulas and relations are presented in Appendices A, B and $\mathrm{Q}$.

\section{THE SCHWINGER-DYSON EQUATIONS IN QED IN A MAGNETIC FIELD.}

The Lagrangian density of massless QED in a magnetic field is

$$
\mathcal{L}=-\frac{1}{4} F^{\mu \nu} F_{\mu \nu}+\frac{1}{2}\left[\bar{\psi},\left(i \gamma^{\mu} D_{\mu}\right) \psi\right]+J^{\mu} A_{\mu}
$$

where the covariant derivative $D_{\mu}$ is

$$
D_{\mu}=\partial_{\mu}-i e A_{\mu}
$$

and the source $J^{\mu}$ provides a chosen external field: $\left\langle 0\left|A_{\mu}\right| 0\right\rangle=A_{\mu}^{\text {ext }}$. As we will see below, in the case of a constant magnetic field $B$, with

$$
A_{\mu}^{e x t}=\left(0,-\frac{B}{2} x_{2}, \frac{B}{2} x_{1}, 0\right),
$$

the source $J^{\mu}=0$. Notice that the vector potential $A_{\mu}^{\text {ext }}(5)$ corresponds to the so called symmetric gauge for the external vector potential, and the magnetic field is in the $+x_{3}$ direction.

Besides the Dirac index $(\mathrm{n})$, the fermion field carries an additional flavor index $a=1,2, \ldots, N$. Then the Lagrangian density in Eq. (3) is invariant under the chiral $S U_{L}(N) \times S U_{R}(N) \times U_{V}(1)$ symmetry (we will discuss the anomalous $U_{A}(1)$ in Sec. VI).

The Schwinger-Dyson (SD) equations in QED in external fields were derived by Schwinger and Fradkin (for a review, see Ref. [17]). The equations for the fermion propagator $G(x, y)$ are

$$
\begin{aligned}
& G^{-1}(x, y)=S^{-1}(x, y)+\Sigma(x, y) \\
& \Sigma(x, y)=4 \pi \alpha \gamma^{\mu} \int G(x, z) \Gamma^{\nu}\left(z, y, z^{\prime}\right) \mathcal{D}_{\nu \mu}\left(z^{\prime}, x\right) d^{4} z d^{4} z^{\prime}
\end{aligned}
$$

Here $S(x, y)$ is the bare fermion propagator in the external field $A_{\mu}^{e x t}, \Sigma(x, y)$ is the fermion mass operator, and $\mathcal{D}_{\mu \nu}(x, y), \Gamma^{\nu}(x, y, z)$ are the full photon propagator and the full amputated vertex.

The full photon propagator satisfies the equations

$$
\begin{aligned}
& \mathcal{D}_{\mu \nu}^{-1}(x, y)=D_{\mu \nu}^{-1}(x-y)+\Pi_{\mu \nu}(x, y), \\
& \Pi_{\mu \nu}(x, y)=-4 \pi \alpha \operatorname{tr} \gamma_{\mu} \int d^{4} u d^{4} z G(x, u) \Gamma_{\nu}(u, z, y) G(z, x),
\end{aligned}
$$

where $D_{\mu \nu}(x-y)$ is the free photon propagator and $\Pi_{\mu \nu}(x, y)$ is the polarization operator.

The equation for the external photon field $A_{\mu}^{e x t}=\left\langle 0\left|A_{\mu}\right| 0\right\rangle$ in a covariant gauge is

$$
\square A_{\mu}^{e x t}-\lambda \partial_{\mu} \partial^{\nu} A_{\nu}^{e x t}=-J_{\mu}-\left\langle 0\left|j_{\mu}\right| 0\right\rangle,
$$

where the vacuum current $\left\langle 0\left|j_{\mu}\right| 0\right\rangle$ is $\left\langle 0\left|j_{\mu}\right| 0\right\rangle=-e \operatorname{tr}\left(\gamma_{\mu} G(x, x)\right)$ and $\lambda$ is the gauge parameter. Notice that for $A_{\mu}^{\text {ext }}$ in Eq. (5), corresponding to a constant magnetic field, the source $J_{\mu}$ is zero. Indeed, in this case, $J_{\mu}=-\left\langle 0\left|j_{\mu}\right| 0\right\rangle$, and $\left\langle 0\left|j_{\mu}\right| 0\right\rangle=-\operatorname{tr}\left(\gamma_{\mu} G(x, x)\right)=0$ because of the symmetry $S O(2) \times S O(1,1)$, with $S O(2)$ and $S O(1,1)$ corresponding to rotations in the $x_{1}-x_{2}$-plane and Lorentz transformations in the $x_{0}-x_{3}$-hyperplane, respectively.

The bare fermion propagator $S(x, y)$ in a constant magnetic field was calculated by Schwinger 18 . In the symmetric gauge (5), it has the form

$$
S(x, y)=\exp \left(i e x^{\mu} A_{\mu}^{e x t}(y)\right) \tilde{S}(x-y)
$$


where the Fourier transform of $\tilde{S}$ is

$$
\begin{aligned}
\tilde{S}(p) & =\int_{0}^{\infty} d s \exp \left[i s\left(p_{0}^{2}-p_{3}^{2}-p_{\perp}^{2} \frac{\tan (e B s)}{e B s}-m\right)\right] \\
& \cdot\left[\left(p^{0} \gamma^{0}-p^{3} \gamma^{3}+m\right)\left(1+\gamma^{1} \gamma^{2} \tan (e B s)\right)-p_{\perp} \gamma_{\perp}\left(1+\tan ^{2}(e B s)\right)\right] .
\end{aligned}
$$

Here a transverse vector $p_{\perp}=\left(p^{1}, p^{2}\right)$ and $m$ is a fermion mass. Then, using the identity

$$
i x^{\mu} A_{\mu}^{e x t}(z)+i z^{\mu} A_{\mu}^{e x t}(y)=i x^{\mu} A_{\mu}^{e x t}(y)+i(x-y)^{\mu} A_{\mu}^{e x t}(z-y)
$$

for the vector potential (5), it is not difficult to show directly from the SD equations (6), (7), (8) and (9) that

$$
\begin{aligned}
& G(x, y)=\exp \left(i e x^{\mu} A_{\mu}^{e x t}(y)\right) \tilde{G}(x-y), \\
& \Gamma(x, y, z)=\exp \left(i e x^{\mu} A_{\mu}^{e x t}(y)\right) \tilde{\Gamma}(x-z, y-z), \\
& \mathcal{D}_{\mu \nu}(x, y)=\tilde{\mathcal{D}}_{\mu \nu}(x-y), \\
& \Pi_{\mu \nu}(x, y)=\tilde{\Pi}_{\mu \nu}(x-y) .
\end{aligned}
$$

In other words, in a constant magnetic field, the Schwinger phase is universal for Green functions containing one fermion field, one antifermion field, and any number of photon fields, and the full photon propagator is translation invariant.

Our aim is to show that there exists a gauge in which the approximation with a bare vertex,

$$
\Gamma^{\mu}(x, y, z)=\gamma^{\mu} \delta(x-y) \delta(x-z),
$$

is reliable for the description of spontaneous chiral symmetry breaking in a magnetic field.

\section{NON-COVARIANT GAUGE AND THE IMPROVED RAINBOW APPROXIMATION FOR THE SD EQUATIONS}

In this section, we will show that there is a (non-covariant) gauge in which the approximation with a bare vertex (15) (the improved rainbow approximation) is reliable.

We begin by recalling the following facts concerning the problem of the magnetic catalysis of chiral symmetry breaking [3]:

1. At weak coupling, there is the LLL dominance in the dynamics of fermion pairing. It is because of the presence of the large Landau gap of order $\sqrt{|e B|}$, which is much larger than the dynamical fermion mass $m_{d y n}$ (for weak coupling). In other words, higher Landau levels decouple from the infrared dynamics (with $k \ll \sqrt{|e B|}$ ) in the same way as it happens with Kaluza-Klein (KK) modes in KK theories of gravity. This fact was explicitly shown in the Nambu-Jona-Lasinio model [3] and in QED [12].

2. The propagator $\tilde{S}(p)(12)$ can be expanded over the Landau levels [19.3]. The contribution from the LLL is

$$
\tilde{S}_{\mathrm{LLL}}(p)=2 i e^{-\left(p_{\perp} l\right)^{2}} \frac{\hat{p}_{\|}+m}{p_{\|}^{2}-m^{2}} O^{(-)},
$$

where the magnetic length $l=|e B|^{-1 / 2}, p_{\perp}=\left(p^{1}, p^{2}\right), p_{\|}=\left(p^{0}, p^{3}\right)$, and $\hat{p}_{\|}=p^{0} \gamma^{0}-p^{3} \gamma^{3}$. The matrix $O^{(-)} \equiv\left(1-i \gamma^{1} \gamma^{2} \operatorname{sign}(e B)\right) / 2$ is the projection operator on the fermion states with the spin polarized along the magnetic field. This point and Eq. (16) clearly reflect the (1+1)-dimensional character of the dynamics of fermions in the LLL. This property is preserved also in the case when the fermion mass is generated dynamically $\left(m=m_{\mathrm{dyn}}\right)$ 卫.

\footnotetext{
${ }^{1}$ As was shown in Refs. [3.20], despite the dimensional reduction $3+1 \rightarrow 1+1$ in the fermion propagator in a magnetic field,
} 
3. The presence of the projection operator $O^{(-)}$implies that the bare vertex for fermions from the LLL is $O^{(-)} \gamma^{\mu} O^{(-)}=O^{(-)} \gamma_{\|}^{\mu}$. Therefore the LLL fermions couple only to the longitudinal $(0,3)$ components of the photon field.

4. In the one-loop approximation, with fermions from the LLL, the photon propagator takes the following form in covariant gauges [3],

$$
\mathcal{D}_{\mu \nu}(q)=-i\left[\frac{1}{q^{2}} g_{\mu \nu}^{\perp}+\frac{q_{\mu}^{\|} q_{\nu}^{\|}}{q^{2} q_{\|}^{2}}+\frac{1}{q^{2}+q_{\|}^{2} \Pi\left(q_{\perp}^{2}, q_{\|}^{2}\right)}\left(g_{\mu \nu}^{\|}-\frac{q_{\mu}^{\|} q_{\nu}^{\|}}{q_{\|}^{2}}\right)-\frac{\lambda}{q^{2}} \frac{q_{\mu} q_{\nu}}{q^{2}}\right],
$$

where the symbols $\perp$ and $\|$ in $g_{\mu \nu}$ are related to the $(1,2)$ and $(0,3)$ components, respectively, and $\lambda$ is a gauge parameter. The explicit expression for $\Pi\left(q_{\perp}^{2}, q_{\|}^{2}\right)=\exp \left[-\left(q_{\perp} l\right)^{2} / 2\right] \Pi\left(q_{\|}^{2}\right)$ is given in Refs. 22, 3]. For our purposes, it is sufficient to know its asymptotes,

$$
\begin{aligned}
& \Pi\left(q_{\|}^{2}\right) \simeq \frac{\bar{\alpha}}{3 \pi} \frac{|e B|}{m_{d y n}^{2}}, \quad \text { as } \quad\left|q_{\|}^{2}\right| \ll m_{d y n}^{2}, \\
& \Pi\left(q_{\|}^{2}\right) \simeq-\frac{2 \bar{\alpha}}{\pi} \frac{|e B|}{q_{\|}^{2}} \quad \text { as } \quad\left|q_{\|}^{2}\right| \gg m_{d y n}^{2},
\end{aligned}
$$

where $\bar{\alpha}=N \alpha$.

Notice that the polarization effects are absent in the transverse components of $\mathcal{D}_{\mu \nu}(q)$. This is because, as was already pointed out in item 3 above, fermions from the LLL couple only to the longitudinal components of the photon field.

5. Then, there is a strong screening effect in the $\left(g_{\mu \nu}^{\|}-q_{\mu}^{\|} q_{\nu}^{\|} / q_{\|}^{2}\right)$ component of the photon propagator. Eq. (19) implies that

$$
\frac{1}{q^{2}+q_{\|}^{2} \Pi\left(q_{\perp}^{2}, q_{\|}^{2}\right)} \simeq \frac{1}{q^{2}-M_{\gamma}^{2}}
$$

with

$$
M_{\gamma}^{2}=\frac{2 \bar{\alpha}}{\pi}|e B|
$$

for $m^{2} \ll\left|q_{\|}^{2}\right| \ll|e B|$ and $\left|q_{\perp}^{2}\right| \ll|e B|$. This is reminiscent of the Higgs effect in the (1+1)-dimensional QED (Schwinger model) 23,24.

We emphasize that the infrared dynamics in this problem is very different from that in the Schwinger model: since photon is neutral, there is no dimensional reduction for its field in a magnetic field, and there is the four-dimensional $q^{2}=q_{\|}^{2}-q_{\perp}^{2}$ in the photon propagator (20). However, the tensor and the spinor structure of this dynamics are exactly the same as in the Schwinger model: indeed, the LLL fermion propagator (16) and the vertex $O^{(-)} \gamma^{\mu} O^{(-)}=O^{(-)} \gamma_{\|}^{\mu}$ are two-dimensional, and only the longitudinal $(0,3)$ components of a photon field are relevant here. This point will be crucial for finding a gauge in which the improved rainbow approximation (with the bare vertex (15)) is reliable 2 .

there is a genuine spontaneous chiral symmetry breaking in this problem: the Mermin-Wagner-Coleman (MWC) theorem [21, forbidding spontaneous breakdown of continuous symmetries at $D=1+1$, is not applicable to this case. The point is that the MWC theorem is based on the fact that gapless Nambu-Goldstone (NG) bosons cannot exist in $1+1$ dimensions. On the other hand, since the NG bosons connected with spontaneous chiral symmetry breaking are neutral, there is no dimensional reduction of their propagator in a magnetic field [3:20]. Therefore their propagator is $(3+1)$-dimensional and there are no obstacles for their existence in this case.

${ }^{2}$ Since an external magnetic field does not lead to confinement of fermions, their mass is gauge invariant in QED in a magnetic field. Therefore any gauge can be used for the calculations of the mass if either the calculations provide the exact result or a good approximation is used: i.e., one can show that corrections to the obtained result are small. Below we will define such a gauge in this model. 
We recall that, as was shown in Ref. [3], despite the smallness of $\alpha$, the expansion in $\alpha$ is broken in covariant gauges in this problem. The reason is that, because of the smallness of $m_{d y n}$ in Eq. (1) as compared to $\sqrt{|e B|}$, there are mass singularities, $\ln \left(|e B| / m_{d y n}^{2}\right) \sim \alpha^{-1 / 2}$, in infrared dynamics. In Appendix $\mathrm{A}$, we analyze these singularities in the vertex. Calculating the one-loop correction to the vertex, one finds that, when external momenta are of order $m_{d y n}$ or less, there are contributions of order $\alpha \ln ^{2}\left(|e B| / m_{d y n}^{2}\right) \sim O(1)$. They come from the term $q_{\mu}^{\|} q_{\nu}^{\|} / q^{2} q_{\|}^{2}$ in $\mathcal{D}_{\mu \nu}(q)$ in Eq. (17).

How can one avoid such mass singularities? A solution is suggested by the Schwinger model. It is known that there is a gauge in which the full vertex is just the bare one [24]. It is the gauge with a bare photon propagator

$$
D_{\alpha \beta}(k)=-i \frac{1}{k^{2}}\left(g_{\alpha \beta}-\frac{k_{\alpha} k_{\beta}}{k^{2}}\right)-i d\left(k^{2}\right) \frac{k_{\alpha} k_{\beta}}{\left(k^{2}\right)^{2}}
$$

with the (non-local) gauge function $d=1 /(1+\Pi)$, where the polarization function $\Pi\left(k^{2}\right)=-e^{2} / \pi k^{2}$ in the Schwinger model (of course, here $\alpha, \beta=0,1$ ). Then, the full propagator is proportional to $g_{\alpha \beta}$,

$$
\mathcal{D}_{\alpha \beta}(k)=D_{\alpha \beta}(k)+i\left(g_{\alpha \beta}-\frac{k_{\alpha} k_{\beta}}{k^{2}}\right) \frac{\Pi\left(k^{2}\right)}{k^{2}\left(1+\Pi\left(k^{2}\right)\right)}=-i \frac{g_{\alpha \beta}}{k^{2}\left(1+\Pi\left(k^{2}\right)\right)} .
$$

The point is that since now $\mathcal{D}_{\alpha \beta}(k) \sim g_{\alpha \beta}$ and since the fermion mass $m=0$ in the Schwinger model, all loop contributions to the vertex are proportional to

$$
P_{2 n+1} \equiv \gamma_{\alpha} \gamma_{\lambda_{1}} \ldots \gamma_{\lambda_{2 n+1}} \gamma^{\alpha}=0
$$

in that gauge and, therefore, disappear.

Let us return to the present problem. As it was emphasized above, the tensor and the spinor structure of the LLL dynamics is $(1+1)$-dimensional. Now, take the bare propagator

$$
D_{\mu \nu}(q)=-i \frac{1}{q^{2}}\left(g_{\mu \nu}-\frac{q_{\mu} q_{\nu}}{q^{2}}\right)-i d\left(q_{\perp}^{2}, q_{\|}^{2}\right) \frac{q_{\mu}^{\|} q_{\nu}^{\|}}{q^{2} q_{\|}^{2}}
$$

with $d=-q_{\|}^{2} \Pi /\left[q^{2}+q_{\|}^{2} \Pi\right]+q_{\|}^{2} / q^{2}$. Then, the full propagator is

$$
\begin{aligned}
\mathcal{D}_{\mu \nu}(q) & =D_{\mu \nu}(q)+i\left(g_{\mu \nu}^{\|}-\frac{q_{\mu}^{\|} q_{\nu}^{\|}}{q_{\|}^{2}}\right) \frac{q_{\|}^{2} \Pi\left(q_{\perp}^{2}, q_{\|}^{2}\right)}{q^{2}\left[q^{2}+q_{\|}^{2} \Pi\left(q_{\perp}^{2}, q_{\|}^{2}\right)\right]} \\
& =-i \frac{g_{\mu \nu}^{\|}}{q^{2}+q_{\|}^{2} \Pi\left(q_{\perp}^{2}, q_{\|}^{2}\right)}-i \frac{g_{\mu \nu}^{\perp}}{q^{2}}+i \frac{q_{\mu}^{\perp} q_{\nu}^{\perp}+q_{\mu}^{\perp} q_{\nu}^{\|}+q_{\mu}^{\|} q_{\nu}^{\perp}}{\left(q^{2}\right)^{2}} .
\end{aligned}
$$

The crucial point is that, as was pointed out above, the transverse degrees of freedom decouple from the LLL dynamics. Therefore only the first term in $\mathcal{D}_{\mu \nu}(q)$, proportional to $g_{\mu \nu}^{\|}$, is relevant.

Notice now that dangerous mass singularities in loop corrections to the vertex might potentially occur only in the terms containing $\hat{q}_{i}^{\|}=q_{i}^{0} \gamma^{0}-q_{i}^{3} \gamma^{3}$ from a numerator $\left(\hat{q}_{i}^{\|}+m_{d y n}\right)$ of each fermion propagator in a diagram (all other terms contain positive powers of $m_{d y n}$, coming from at least some of the numerators and, therefore, are harmless). However, because of the same reasons as in the gauge (23) in the Schwinger model, all those potentially dangerous terms disappear in the gauge (26). Therefore all the loop corrections to the vertex are suppressed by positive powers of $\alpha$ in this gauge. This in turn implies that those loop corrections may result only in a change $\tilde{C} \sim O(1) \rightarrow \tilde{C}^{\prime} \sim O(1)$ in expression (2.) In other words, in gauge (26) there exists a consistent truncation of the SD equations and the problem is essentially soluble in that gauge.

In the next section, we will consider the loop expansion for the SD equations in this problem in more detail.

\footnotetext{
${ }^{3} P_{2 n+1}=0$ follows from the two identities for the two-dimensional Dirac matrices: $\gamma_{\alpha} \gamma_{\lambda} \gamma^{\alpha}=0$ and $\gamma_{\lambda_{i}} \gamma_{\lambda_{i+1}}=g_{\lambda_{i} \lambda_{i+1}}+$ $\varepsilon_{\lambda_{i} \lambda_{i+1}} \gamma_{5}\left(\gamma_{5}=\gamma_{0} \gamma_{1}, \varepsilon_{\alpha \beta}=-\varepsilon_{\beta \alpha}, \varepsilon_{01}=1\right)$.

${ }^{4}$ The gauge (26) is unique in that. In other gauges, there is an infinite set of diagrams giving relevant contributions to the vertex. Therefore, in other gauges, one needs to sum up an infinite set of diagrams to recover the same result for the fermion mass.
} 


\section{THE LOOP EXPANSION FOR THE SD EQUATIONS}

The consideration of mass singularities in loop corrections given at the end of the previous section was, though general, somewhat heuristic. First of all, one has to define more rigorously the perturbative expansion for the SD equations which is used in this problem. It is the loop expansion based on the CJT (Cornwall, Jackiw, and Tomboulis) effective action $\Gamma\left(G, \mathcal{D}_{\mu \nu}\right)$ for composite operators 25] (for a review see Ref. 26]). The conditions for extrema of $\Gamma$ yield the SD equations,

$$
\frac{\delta \Gamma}{\delta G(x, y)}=0, \quad \frac{\delta \Gamma}{\delta \mathcal{D}_{\mu \nu}(x, y)}=0 .
$$

In the loop expansion for $\Gamma$, the full photon and fermion propagators are used in two-particle irreducible diagrams for $\Gamma$. In QED, the problem is essentially reduced to the loop expansion (with the full photon and fermion propagators) for the vertex.

The full photon propagator is given by Eq. (26), and the full propagator for fermions from the LLL has the form

$$
\tilde{G}(p)=2 i e^{-\left(p_{\perp} l\right)^{2}} \frac{A\left(p_{\|}^{2}\right) \hat{p}_{\|}+B\left(p_{\|}^{2}\right)}{A^{2}\left(p_{\|}^{2}\right) p_{\|}^{2}-B^{2}\left(p_{\|}^{2}\right)} O^{(-)}
$$

(compare with Eq. (16) and see below). Here $B\left(p_{\|}^{2}\right)$ is a dynamical mass function of fermions.

In this section we will derive the SD equations for the fermion propagator in the one-loop and two-loop approximations. It will be shown, that while the one-loop approximation, coinciding with the improved rainbow approximation, is reliable in non-covariant gauge (26), it is not reliable in covariant gauges (17).

From Eqs. (6) and (7) one gets the following equation for the fermion propagator $G(x, y)$ in the two-loop approximation (by using the vertex function in the one-loop approximation, see Eq. (14b) and Eq. (A1) in Appendix A),

$$
\begin{aligned}
& G(x, y)=S(x, y)-4 \pi \alpha \int d^{4} x_{1} d^{4} y_{1} S\left(x, x_{1}\right) \gamma^{\mu} G\left(x_{1}, y_{1}\right) \gamma^{\nu} G\left(y_{1}, y\right) \mathcal{D}_{\mu \nu}\left(x_{1}-y_{1}\right) \\
& +(4 \pi \alpha)^{2} \int d^{4} x_{1} d^{4} x_{2} d^{4} y_{1} d^{4} y_{2} S\left(x, x_{1}\right) \gamma^{\mu} G\left(x_{1}, x_{2}\right) \gamma^{\sigma} G\left(x_{2}, y_{2}\right) \gamma^{\nu} G\left(y_{2}, y_{1}\right) \gamma^{\rho} G\left(y_{1}, y\right) \mathcal{D}_{\mu \nu}\left(x_{1}-y_{2}\right) \mathcal{D}_{\sigma \rho}\left(x_{2}-y_{1}\right)
\end{aligned}
$$

Here $S(x, y)$ is the bare fermion propagator of massless fermions $(m=0)$. The graphic form of this equation is shown in Fig. 1. After extracting the Schwinger phase factors in the full and bare fermion propagators [see Eq. (14a)],

$$
G(x, y)=e^{i x^{\nu} A_{\nu}^{e x t}(y)} \tilde{G}(x-y), \quad S(x, y)=e^{i x^{\nu} A_{\nu}^{e x t}(y)} \tilde{S}(x-y),
$$

Eq. (29) reads as

$$
\begin{aligned}
\tilde{G}(x)= & \tilde{S}(x)-4 \pi \alpha \int d^{4} x_{1} d^{4} y_{1} e^{i x A\left(x_{1}\right)+i x_{1} A\left(y_{1}\right)} \tilde{S}\left(x-x_{1}\right) \gamma^{\mu} \tilde{G}\left(x_{1}-y_{1}\right) \gamma^{\nu} \tilde{G}\left(y_{1}\right) \mathcal{D}_{\mu \nu}\left(x_{1}-y_{1}\right) \\
+ & (4 \pi \alpha)^{2} \int d^{4} x_{1} d^{4} x_{2} d^{4} y_{1} d^{4} y_{2} e^{i x A\left(x_{1}\right)+i x_{1} A\left(x_{2}\right)+i x_{2} A\left(y_{2}\right)+i y_{2} A\left(y_{1}\right)} \\
& \times \tilde{S}\left(x-x_{1}\right) \gamma^{\mu} \tilde{G}\left(x_{1}-x_{2}\right) \gamma^{\sigma} \tilde{G}\left(x_{2}-y_{2}\right) \gamma^{\nu} \tilde{G}\left(y_{2}-y_{1}\right) \gamma^{\rho} \tilde{G}\left(y_{1}\right) \mathcal{D}_{\mu \nu}\left(x_{1}-y_{2}\right) \mathcal{D}_{\sigma \rho}\left(x_{2}-y_{1}\right)
\end{aligned}
$$

where $A_{\mu}^{\text {ext }}$ is given in Eq. (5) and the shorthand $x A^{\text {ext }}(y)$ stands for $x^{\mu} A_{\mu}^{e x t}(y)$.

First, let us show that the solution to the above equation, $\tilde{G}(x)$, allows the factorization of the dependence on the parallel and perpendicular coordinates,

$$
\tilde{G}(x)=\frac{i}{2 \pi l^{2}} \exp \left(-\frac{x_{\perp}^{2}}{4 l^{2}}\right) g\left(x_{\|}\right) O^{(-)} .
$$

Notice that this form for $\tilde{G}(x)$ is suggested by a similar expression for the bare propagator,

$$
\tilde{S}(x)=\frac{i}{2 \pi l^{2}} \exp \left(-\frac{x_{\perp}^{2}}{4 l^{2}}\right) s\left(x_{\|}\right) O^{(-)},
$$

with 


$$
s\left(x_{\|}\right)=\int \frac{d^{2} k_{\|}}{(2 \pi)^{2}} e^{-i k_{\|} x_{\|}} \frac{\hat{k}_{\|}+m}{k_{\|}^{2}-m^{2}}
$$

(see Eq. (16); in the chiral limit, as in the present problem, the bare mass $m=0$ ). In order to perform the integrations over the perpendicular components of $x_{1}$ and $y_{1}$ in Eq. (31), it is convenient to make use of the photon propagator in the momentum representation,

$$
\mathcal{D}_{\mu \nu}(x)=\int \frac{d^{2} q_{\|} d^{2} q_{\perp}}{(2 \pi)^{4}} e^{-i q_{\|} x_{\|}+i q_{\perp} x_{\perp}} \mathcal{D}_{\mu \nu}\left(q_{\|}, q_{\perp}\right) .
$$

After substituting this representation along with those in Eqs. (32) and (33) into the SD equation (31) and performing the straightforward, though tedious, integrations over $x_{1}^{\perp}, y_{1}^{\perp}, x_{2}^{\perp}$ and $y_{2}^{\perp}$ we arrive at

$$
\begin{aligned}
& g\left(x_{\|}\right)=s\left(x_{\|}\right)+4 \pi \alpha \int \frac{d^{4} q}{(2 \pi)^{4}} d^{2} x_{1}^{\|} d^{2} y_{1}^{\|} \exp \left(-\frac{\left(q_{\perp} l\right)^{2}}{2}-i q_{\|}\left(x_{1}^{\|}-y_{1}^{\|}\right)\right) s\left(x^{\|}-x_{1}^{\|}\right) \gamma_{\|}^{\mu} g\left(x_{1}^{\|}-y_{1}^{\|}\right) \gamma_{\|}^{\nu} g\left(y_{1}^{\|}\right) \mathcal{D}_{\mu \nu}\left(q_{\|}, q_{\perp}\right) \\
& +(4 \pi \alpha)^{2} \int \frac{d^{4} q_{1} d^{4} q_{2}}{(2 \pi)^{8}} d^{2} x_{1}^{\|} d^{2} y_{1}^{\|} d^{2} x_{2}^{\|} d^{2} y_{2}^{\|} \exp \left(-\frac{\left(q_{1}^{\perp} l\right)^{2}}{2}-\frac{\left(q_{2}^{\perp} l\right)^{2}}{2}+i l^{2}\left[q_{1}^{\perp} \times q_{2}^{\perp}\right]-i q_{1}^{\|}\left(x_{1}^{\|}-y_{2}^{\|}\right)-i q_{2}^{\|}\left(x_{2}^{\|}-y_{1}^{\|}\right)\right) \\
& \times s\left(x^{\|}-x_{1}^{\|}\right) \gamma_{\|}^{\mu} g\left(x_{1}^{\|}-x_{2}^{\|}\right) \gamma_{\|}^{\sigma} g\left(x_{2}^{\|}-y_{2}^{\|}\right) \gamma_{\|}^{\nu} g\left(y_{2}^{\|}-y_{1}^{\|}\right) \gamma_{\|}^{\rho} g\left(y_{1}^{\|}\right) \mathcal{D}_{\mu \nu}\left(q_{1}^{\|}, q_{1}^{\perp}\right) \mathcal{D}_{\sigma \rho}\left(q_{2}^{\|}, q_{2}^{\perp}\right),
\end{aligned}
$$

where $\left[q_{1}^{\perp} \times q_{2}^{\perp}\right] \equiv \epsilon_{3 i j} q_{1 i} q_{2 j}$. Since no dependence on $x_{\perp}$ has left, we conclude that the form of $\tilde{G}(x)$ in Eq. (32) is indeed consistent with the structure of the SD equation.

Regarding this equation, it is necessary to emphasize that the "perpendicular" components of the $\gamma$-matrices are absent in it. Indeed, because of the identity $O^{(-)} \gamma_{\perp}^{\mu} O^{(-)}=0$, all those components are killed by the projection operators coming from the fermion propagators.

Substituting now the photon propagator in the Feynman-like (non-covariant) gauge (26) into the SD equation, we see that only the first term in Eq. (26), proportional to $g_{\mu \nu}^{\|}$, leads to a nonvanishing contribution. In other words, the photon propagator is effectively proportional to $g_{\mu \nu}^{\|}$(justifying the name of the gauge).

By switching to the momentum space, we obtain

$$
\begin{aligned}
& g^{-1}\left(p_{\|}\right)=s^{-1}\left(p_{\|}\right)-4 \pi \alpha \int \frac{d^{4} q}{(2 \pi)^{4}} \exp \left(-\frac{\left(q_{\perp} l\right)^{2}}{2}\right) \gamma_{\|}^{\mu} g\left(p^{\|}-q^{\|}\right) \gamma_{\|}^{\nu} \mathcal{D}_{\mu \nu}\left(q_{\|}, q_{\perp}\right) \\
& -(4 \pi \alpha)^{2} \int \frac{d^{4} q_{1} d^{4} q_{2}}{(2 \pi)^{8}} \exp \left(-\frac{\left(q_{1}^{\perp} l\right)^{2}}{2}-\frac{\left(q_{2}^{\perp} l\right)^{2}}{2}+i l^{2}\left[q_{1}^{\perp} \times q_{2}^{\perp}\right]\right) \\
& \times \gamma_{\|}^{\mu} g\left(p^{\|}-q_{1}^{\|}\right) \gamma_{\|}^{\sigma} g\left(p^{\|}-q_{1}^{\|}-q_{2}^{\|}\right) \gamma_{\|}^{\nu} g\left(p^{\|}-q_{2}^{\|}\right) \gamma_{\|}^{\rho} \mathcal{D}_{\mu \nu}\left(q_{1}^{\|}, q_{1}^{\perp}\right) \mathcal{D}_{\sigma \rho}\left(q_{2}^{\|}, q_{2}^{\perp}\right) .
\end{aligned}
$$

The general solution to this equation is given by the ansatz,

$$
g\left(p_{\|}\right)=\frac{A_{p} \hat{p}_{\|}+B_{p}}{A_{p}^{2} p_{\|}^{2}-B_{p}^{2}}
$$

where $A_{p}=A\left(p_{\|}^{2}\right)$ and $B_{p}=B\left(p_{\|}^{2}\right)$. Making use of this as well as of the explicit form of the photon propagator, the previous equation splits into the system of two coupled equations,

$$
\begin{aligned}
A_{p}= & +\left(\frac{\alpha}{2 \pi^{3}}\right)^{2} \int \frac{d^{2} q_{1}^{\|} d^{2} q_{2}^{\|} B_{p-q_{1}} A_{p-q_{1}-q_{2}} B_{p-q_{2}}}{\left(A_{p-q_{1}}^{2}\left(p^{\|}-q_{1}^{\|}\right)^{2}-B_{p-q_{1}}^{2}\right)\left(A_{p-q_{1}-q_{2}}^{2}\left(p^{\|}-q_{1}^{\|}-q_{2}^{\|}\right)^{2}-B_{p-q_{1}-q_{2}}^{2}\right)\left(A_{p-q_{2}}^{2}\left(p^{\|}-q_{2}^{\|}\right)^{2}-B_{p-q_{2}}^{2}\right)} \\
& \times \int \frac{d^{2} q_{1}^{\perp} d^{2} q_{2}^{\perp} \exp \left(-\left(q_{1}^{\perp} l\right)^{2} / 2-\left(q_{2}^{\perp} l\right)^{2} / 2+i l^{2}\left[q_{1}^{\perp} \times q_{2}^{\perp}\right]\right)}{\left[q_{1}^{2}+\left(q_{1}^{\|}\right)^{2} \Pi\left(\left(q_{1}^{\perp}\right)^{2},\left(q_{1}^{\|}\right)^{2}\right)\right]\left[q_{2}^{2}+\left(q_{2}^{\|}\right)^{2} \Pi\left(\left(q_{2}^{\perp}\right)^{2},\left(q_{2}^{\|}\right)^{2}\right)\right]}, \\
B_{p}= & -i \frac{\alpha}{2 \pi^{3}} \int \frac{d^{2} q_{\|} B_{p-q}}{\left(p_{\|}-q_{\|}\right)^{2}-B_{p-q}^{2}} \int \frac{d^{2} q_{\perp} \exp \left(-\left(q_{\perp} l\right)^{2} / 2\right)}{q^{2}+q_{\|}^{2} \Pi\left(q_{\perp}^{2}, q_{\|}^{2}\right)} \\
& -\left(\frac{\alpha}{2 \pi^{3}}\right)^{2} \int \frac{d^{2} q_{1}^{\|} d^{2} q_{2}^{\|}\left(p_{\|}^{2}+q_{1}^{\|} \cdot q_{2}^{\|}\right) A_{p-q_{1}} B_{p-q_{1}-q_{2}} A_{p-q_{2}}}{\left(A_{p-q_{1}}^{2}\left(p^{\|}-q_{1}^{\|}\right)^{2}-B_{p-q_{1}}^{2}\right)\left(A_{p-q_{1}-q_{2}}^{2}\left(p^{\|}-q_{1}^{\|}-q_{2}^{\|}\right)^{2}-B_{p-q_{1}-q_{2}}^{2}\right)\left(A_{p-q_{2}}^{2}\left(p^{\|}-q_{2}^{\|}\right)^{2}-B_{p-q_{2}}^{2}\right)} \\
& \times \int \frac{d^{2} q_{1}^{\perp} d^{2} q_{2}^{\perp} \exp \left(-\left(q_{1}^{\perp} l\right)^{2} / 2-\left(q_{2}^{\perp} l\right)^{2} / 2+i l^{2}\left[q_{1}^{\perp} \times q_{2}^{\perp}\right]\right)}{\left[q_{1}^{2}+\left(q_{1}^{\|}\right)^{2} \Pi\left(\left(q_{1}^{\perp}\right)^{2},\left(q_{1}^{\|}\right)^{2}\right)\right]\left[q_{2}^{2}+\left(q_{2}^{\|}\right)^{2} \Pi\left(\left(q_{2}^{\perp}\right)^{2},\left(q_{2}^{\|}\right)^{2}\right)\right]} .
\end{aligned}
$$


This concludes our derivation of the two-loop SD equations for $A_{p}$ and $B_{p}$ which define the fermion propagator through Eq. (38).

Let us now show that the improved rainbow (one-loop) approximation is reliable in gauge (26). In that approximation, the SD equations (39) and (40) are

$$
\begin{aligned}
& A_{p}=1, \\
& B_{p}=-i \frac{\alpha}{2 \pi^{3}} \int \frac{d^{2} q_{\|} B_{p-q}}{\left(p_{\|}-q_{\|}\right)^{2}-B_{p-q}^{2}} \int \frac{d^{2} q_{\perp} \exp \left(-\left(q_{\perp} l\right)^{2} / 2\right)}{q^{2}+q_{\|}^{2} \Pi\left(q_{\perp}^{2}, q_{\|}^{2}\right)} .
\end{aligned}
$$

Equation (42) was solved both numerically and analytically (see the next section and Appendix B). In particular, it was shown that the approximation with $B\left(p_{\|}^{2}\right)=m_{\mathrm{dyn}}$ for $p_{\|}^{2}<2|e B|$ and $B\left(p_{\|}^{2}\right)$ rapidly decreasing for $p_{\|}^{2}>2|e B|$ is a very good one (see Fig. 3 in the next Section). Moreover, as it is shown in Sec.V and Appendix B, in the improved rainbow approximation, it is sufficient to use the constant photon mass approximation for the polarization function: $q_{\|}^{2} \Pi\left(q_{\perp}^{2}, q_{\|}^{2}\right) \simeq-M_{\gamma}^{2} \equiv-2 \bar{\alpha}|e B| / \pi$ [see Eq. (19)]. Then, equation (40) at $p_{\|}=0$ reduces to

$$
1 \simeq \frac{\alpha}{4 \pi} \ln \left(\frac{1}{\left(M_{\gamma} l\right)^{2}}\right) \ln \left(\frac{M_{\gamma}^{2}}{m_{d y n}^{2}\left(m_{d y n} l\right)^{2}}\right)+\operatorname{Const} \frac{\alpha^{2}}{8 \pi^{2}} \ln ^{2}\left(\frac{1}{\left(M_{\gamma} l\right)^{2}}\right) \ln \left(\frac{M_{\gamma}^{2}}{m_{d y n}^{2}\left(m_{d y n} l\right)^{2}}\right),
$$

where the two terms in the right hand side come out as the estimates of the following two integrals,

$$
\begin{aligned}
I_{1}= & \frac{\alpha}{2 \pi} \int_{0}^{\infty} \frac{d x d y \exp \left(-x\left(m_{d y n} l\right)^{2} / 2\right)}{(y+1)\left(x+y+\left(M_{\gamma} / m_{d y n}\right)^{2}\right)}, \\
I_{2}= & \frac{\alpha^{2}}{8 \pi^{2}} \int_{0}^{\infty} \frac{d x_{1} d y_{1} d x_{2} d y_{2} \exp \left(-x_{1}\left(m_{d y n} l\right)^{2} / 2-x_{2}\left(m_{d y n} l\right)^{2} / 2\right) J_{0}\left(\left(m_{d y n} l\right)^{2} \sqrt{x_{1} x_{2}}\right)}{\left(y_{1}+1\right)\left(y_{2}+1\right)\left(x_{1}+y_{1}+\left(M_{\gamma} / m_{d y n}\right)^{2}\right)\left(x_{2}+y_{2}+\left(M_{\gamma} / m_{d y n}\right)^{2}\right)} \\
& \times\left(\frac{y_{1}+y_{2}+1}{\sqrt{\left(y_{1}-y_{2}\right)^{2}+2\left(y_{1}+y_{2}\right)+1}}-1\right) .
\end{aligned}
$$

By assuming that the first term on the right hand side in Eq. (43) is of order one, we see that the second term, corresponding to the two-loop correction, is indeed suppressed: it is of order $\alpha \log 1 / \alpha$. Note that we should have expected this result taking into account the calculations in Appendix A. As is shown there, the higher order corrections to the vertex are suppressed in the Feynman-like gauge, and the two-loop contribution in the SD equation occurs as a result of the one-loop insertion in the vertex (see Fig. 1).

If we repeat the same analysis in the case of the covariant gauge (17), we end up with the following estimate,

$$
1 \simeq \frac{\alpha}{4 \pi} \ln ^{2}\left(\frac{1}{\left(m_{d y n} l\right)^{2}}\right)+\text { Const } \alpha^{2} \ln ^{4}\left(\frac{1}{\left(m_{d y n} l\right)^{2}}\right)
$$

where the main contribution to both terms, as is easy to check, comes from the $q_{\|}^{\mu} q_{\|}^{\mu} / q^{2} q_{\|}^{2}$ component in the photon propagator. In contrast to what we had in the Feynman-like gauge, after assuming that the first term in Eq. (46) is of order of one, we see that the second term would be also of order one. In other words, there is no suppression of higher order terms in the covariant gauge. Similarly, there is no suppression in all other gauges in which the $q_{\|}^{\mu} q_{\|}^{\mu} / q^{2} q_{\|}^{2}$ component is non-zero. Therefore, in agreement with the general arguments of the previous section, the Feynman-like gauge (26) is special: in this gauge there exists a consistent truncation of the SD equations.

Since the dynamical mass is a gauge invariant quantity, in other gauges one needs to sum up an infinite set of diagrams to recover the same result for it. Obviously it is rather difficult to classify all the relevant diagrams in those gauges, and so the existence of the Feynman-like gauge (26) is the key point that allows to treat the present problem reliably.

\section{THE ANALYSIS OF THE SD EQUATIONS}

As was shown in the previous section, in the gauge (26), the SD equations (6), (7), (8) and (9) with the bare vertex (15) are reliable. They form a closed system of integral equations. In Euclidean space Eq. (42) has the form 


$$
B\left(p_{\|}^{2}\right)=\frac{\alpha}{2 \pi^{2}} \int \frac{d^{2} q_{\|} B\left(\left(p_{\|}-q_{\|}\right)^{2}\right)}{\left(p_{\|}-q_{\|}\right)^{2}+B^{2}\left(\left(p_{\|}-q_{\|}\right)^{2}\right)} \int_{0}^{\infty} \frac{d x \exp \left(-x l^{2} / 2\right)}{x+q_{\|}^{2}+q_{\|}^{2} \Pi_{E}\left(x, q_{\|}^{2}\right)},
$$

where the polarization function $\Pi_{E}\left(q_{\perp}^{2}, q_{\|}^{2}\right)$ is defined from Eq. (9) with the bare vertex (15).

Equation (47) was solved by using both analytical and numerical methods. The analytical approach is considered in Appendix B. Here we will describe the numerical solution.

The results of the numerical analysis of Eq. (47) are shown in Figs. 2 , 3 and 4 . The crucial points in the analysis are the following:

a) The polarization function $\Pi_{E}\left(q_{\perp}^{2}, q_{\|}^{2}\right)$ is in principle a complicated functional of the fermion mass function $B\left(p_{\|}^{2}\right)$. However, as it is shown in Appendix B, the leading singularity, $1 / \alpha \ln (\alpha)$, in $\ln \left(m_{d y n}^{2}\right)$ in Eq. (2) is induced in the kinematic region with $m_{d y n}^{2} \ll\left|q_{\|}^{2}\right| \ll|e B|$ and $m_{d y n}^{2} \ll M_{\gamma}^{2} \lesssim q_{\perp}^{2} \ll|e B|$. In that region, the fermions can be treated as massless, and therefore the polarization function is $\Pi_{E}\left(q_{\perp}^{2}, q_{\|}^{2}\right) \simeq 2 \bar{\alpha}|e B| / \pi q_{\|}^{2}=M_{\gamma}^{2} / q_{\|}^{2}$ [see Eqs. (19) and (21)]. In other words, in this approximation, the photon propagator is a propagator of a free massive boson with $M_{\gamma}^{2}=2 \bar{\alpha}|e B| / \pi$.

b) In the numerical solution, the following ansatz for $\ln \left(m_{d y n}\right)$ was used,

$$
\ln \frac{m_{d y n}}{\sqrt{2|e B|}}=\ln a_{0}+\frac{a_{1}}{3} \ln \frac{N \alpha}{\pi}-\frac{a_{2}}{\left(\frac{\alpha}{\pi}\right)^{a_{3}} \ln ^{a_{4}} \frac{a_{5} \pi}{N \alpha} .} .
$$

For small $\alpha(0.001 \leq \alpha \leq 0.1)$ and different $N(1 \leq N \leq 7)$ the best fit was found with $a_{0}=a_{1}=a_{2}=a_{3}=a_{4}=1$ and $a_{5} \simeq 0.58 \pm 0.02$ (see Fig. $\mathbb{1}$ ). This fit corresponds to the expression

$$
m_{d y n}=\tilde{C} \sqrt{|e B|} F(\alpha) \exp \left[-\frac{\pi}{\alpha \ln \left(C_{1} / N \alpha\right)}\right]
$$

where $F(\alpha) \simeq(N \alpha)^{1 / 3}, C_{1} \simeq 1.82 \pm 0.06$ and $\tilde{C}=\sqrt{2}$.

c) The numerical solution shows that the function $B\left(p_{\|}^{2}\right)$ is essentially constant for $p_{\|}^{2} \ll|e B|, B\left(p_{\|}^{2}\right)=m_{d y n}$, and rapidly decreases for $p_{\|}^{2} \gg|e B|$, see Fig. 3. Therefore this approximation is self-consistent: the Ward-Takahashi identity for the vertex is satisfied in the relevant kinematic region of momenta (see Appendix (C), and the pole of the fermion propagator appears at $p_{\|}^{2}=m_{d y n}^{2}$.

It is instructive to clarify the origin of the difference between the dynamical mass $m_{d y n}$ (11), calculated in the rainbow approximation, and the expression for $m_{d y n}$ (49). The integral equation (47) for $B\left(p_{\|}^{2}\right)$ can be rewritten in the form,

$$
B\left(p^{2}\right)=\frac{\alpha}{2 \pi^{2}} \int \frac{d^{2} q B\left(q^{2}\right)}{q^{2}+B^{2}\left(q^{2}\right)} \int_{0}^{\infty} \frac{d x \exp \left(-x l^{2} / 2\right)}{x+(\mathbf{q}-\mathbf{p})^{2}+M_{\gamma}^{2}},
$$

with $M_{\gamma}^{2}=0$ and $M_{\gamma}^{2}=2 \bar{\alpha}|e B| / \pi$ in the rainbow approximation and in the improved rainbow approximation, in gauge (26), respectively (henceforth we will omit the symbol $\|$; the two-dimensional vector $\left.\mathbf{q}=\left(q_{4}, q_{3}\right), q_{4}=-i q_{0}\right)$.

The numerical analysis of this integral equation shows that the so called linearized approximation, with $B^{2}\left(q^{2}\right) \rightarrow$ $m_{\mathrm{dyn}}^{2}=B^{2}(0)$ in the denominator of Eq. (50), is an excellent approximation. Then we get

$$
B\left(p^{2}\right)=\frac{\alpha}{2 \pi^{2}} \int \frac{d^{2} q B\left(q^{2}\right)}{q^{2}+m_{d y n}^{2}} \int_{0}^{\infty} \frac{d x \exp \left(-x l^{2} / 2\right)}{x+(\mathbf{q}-\mathbf{p})^{2}+M_{\gamma}^{2}} .
$$

This equation is equivalent to a two-dimensional Schrödinger-like differential equation. Indeed, introducing the function

$$
\Psi(\mathbf{r})=\int \frac{d^{2} q}{(2 \pi)^{2}} \frac{B\left(q^{2}\right)}{q^{2}+m_{d y n}^{2}} e^{i \mathbf{q r}},
$$

we get the equation

$$
\left(-\Delta+m_{d y n}^{2}+V(\mathbf{r})\right) \Psi(\mathbf{r})=0
$$


where $\Delta=\partial^{2} / \partial x_{3}^{2}+\partial^{2} / \partial x_{4}^{2}$ and the potential $V(\mathbf{r})$ is

$$
\begin{aligned}
V(\mathbf{r}) & =-\frac{\alpha}{2 \pi^{2}} \int^{2} d^{2} e^{i \mathbf{p r}} \int_{0}^{\infty} \frac{d x \exp (-x / 2)}{l^{2} p^{2}+x+l^{2} M_{\gamma}^{2}} \\
& =-\frac{\alpha}{\pi l^{2}} \int_{0}^{\infty} d x e^{-x / 2} K_{0}\left(\frac{r}{l} \sqrt{x+l^{2} M_{\gamma}^{2}}\right) .
\end{aligned}
$$

At $M_{\gamma}^{2}=0$ (the rainbow approximation), the last integral is expressed through the integral exponential function $E i(x)=-\int_{-x}^{\infty} d t \exp (-t) / t$,

$$
\left.V_{0}(\mathbf{r}) \equiv V(\mathbf{r})\right|_{M_{\gamma}=0}=\frac{\alpha}{\pi l^{2}} \exp \left(\frac{r^{2}}{2 l^{2}}\right) E i\left(-\frac{r^{2}}{2 l^{2}}\right) .
$$

Using the asymptotic relations for $\operatorname{Ei}(x)$ [32], we get

$$
\begin{aligned}
& V_{0}(\mathbf{r}) \simeq-\frac{2 \alpha}{\pi} \frac{1}{r^{2}}, \quad r \gg l \\
& V_{0}(\mathbf{r}) \simeq-\frac{\alpha}{\pi l^{2}} \log \frac{2 l^{2}}{r^{2}}, \quad r \ll l .
\end{aligned}
$$

Equation (56a) implies that the potential is long-range in the rainbow approximation.

Using now the asymptotic relations for $K_{0}(z)$ [32], we get the following asymptotes for $V(\mathbf{r})$ at $M_{\gamma}^{2} \neq 0$,

$$
\begin{aligned}
& V(\mathbf{r}) \simeq-\sqrt{\frac{2}{\pi}} \alpha \frac{M_{\gamma}^{1 / 2}}{r^{3 / 2}} e^{-M_{\gamma} r}, \quad r \gg \frac{1}{M_{\gamma}} \gg l, \\
& V(\mathbf{r}) \simeq-\frac{\alpha}{\pi l^{2}} \log \frac{2 l^{2}}{r^{2}}, \quad r \ll l .
\end{aligned}
$$

Therefore, while the short-distance behavior of the potential is independent of $M_{\gamma}$ (compare Eqs. (56b) and (57b)), its long-distance behavior in the rainbow approximation and in the improved rainbow approximation is essentially different: while the former potential is long-range [see Eq. (56a)], the latter is short-range [see Eq. (57a)]. This point yields the physical origin of the difference of expressions (11) and (49) for $m_{d y n}$ in those two approximations.

It is also instructive to discuss this point using results proved in the literature for the two-dimensional $(d=2)$ Schrödinger equation [31]. The form of equation (53) implies that $-m_{d y n}^{2}$ plays the role of energy $E$ in the Schrödinger equation. The results of Ref. [31] ensure that a) there is at least one bound state for any attractive potential for $d=2$, i.e., there is at least one solution with $m_{\mathrm{dyn}}^{2}=-E>0$; b) for short-range potentials of the form $V(\mathbf{r})=\alpha \tilde{V}(\mathbf{r})$, where $\tilde{V}(\mathbf{r})$ is independent of $\alpha$, the energy $E=-m_{d y n}^{2}$ of the lowest bound state is $-E(\alpha)=m_{\text {dyn }}^{2}(\alpha) \sim \exp (-1 / a \alpha)$, with $a>0$, as $\alpha \rightarrow 0$. If the mass $M_{\gamma}$ were independent of $\alpha$, the potential (57a) would satisfy the constraints in item b) above. However, because $M_{\gamma}^{2}=2 N \alpha|e B| / \pi \rightarrow 0$ as $\alpha \rightarrow 0$, we get an additional logarithmic factor in the power of the exponent in equation (49). On the other hand, since the potential $V(\mathbf{r})$ is long-range in the rainbow approximation [see Eq. (56a)], it clearly does not satisfy the constraints in item b) above, and $m_{d y n}^{2} \sim \exp (-1 / a \sqrt{\alpha})$ in that approximation. $]^{5}$

\section{CONCLUSION}

The magnetic catalysis of chiral symmetry breaking in QED is a phenomenon with rather rich and sophisticated dynamics. It yields a (first, to the best of our knowledge) example in which there exists a consistent truncation of

\footnotetext{
${ }^{5}$ As follows from the analysis in Appendix B, $m_{d y n}$ would be indeed $m_{d y n} \sim \sqrt{|e B|} \exp (-1 / a \alpha)$, with $a \sim \log |e B| / M_{\gamma}^{2}$, in the case of $M_{\gamma}^{2}$ independent of $\alpha$. Notice that the transition from $M_{\gamma}^{2}=2 N \alpha|e B| / \pi$ (the improved rainbow approximation) to $M_{\gamma}^{2}=0$ (the rainbow approximation) corresponds to changing $a \sim \log |e B| / M_{\gamma}^{2} \sim \log 1 / N \alpha$ to $a \sim \log |e B| / m_{d y n}^{2}$. Then, expression (49) for $m_{d y n}$ transforms into expression (14).
} 
the Schwinger-Dyson equations in the problem of dynamical symmetry breaking in a (3+1)-dimensional gauge theory without fundamental scalar fields.

It is instructive to compare this problem with dynamical symmetry breaking in $(3+1)$-dimensional gauge theories without external fields (for a review, see Refs. [26,27). In the case of non-Abelian gauge theories, such as QCD, dynamical chiral symmetry breaking is generated in the infrared region, where the effective coupling constant is strong. This prevents to elaborate a consistent truncation of the Schwinger-Dyson equations in those theories. In Abelian gauge theories, on the other hand, a solution with a non-zero fermion mass exists in ladder approximation for any value of the coupling constant [28], if there is no ultraviolet cutoff in the Schwinger-Dyson equations (at finite cutoff, in the ladder approximation, dynamical chiral symmetry breaking takes place only if the coupling constant is large enough [26]). This fact implies that the ultraviolet region is responsible for chiral symmetry breaking in that case. Since the running coupling is strong in the ultraviolet region in Abelian gauge theories, this again prevents to elaborate a consistent truncation of the Schwinger-Dyson equations in those theories either. In contrast, the dynamics of chiral symmetry breaking in QED in a magnetic field is long-range, and the QED coupling constant is small in infrared. As a result, a consistent truncation of the SD equations exists in the present problem in the gauge (26).

The crucial point in the present analysis is the dimensional reduction in the dynamics of the fermion pairing. However, there is an essential difference between QED and the Nambu-Jona-Lasinio model in a magnetic field: in QED, there is an additional neutral field, the photon field. As a result, the dynamics in QED in a magnetic field is much more sophisticated than that in the NJL model. In particular, the photon propagator includes the fourdimensional $q^{2}=q_{\|}^{2}-q_{\perp}^{2}$ and is not reduced to the two-dimensional form. However, the tensor and the spinor structure in this model in the infrared region is exactly the same as in the Schwinger model. This point is crucial for making this problem essentially soluble.

What is the chiral symmetry, $S U_{L}(N) \times S U_{R}(N) \times U_{V}(1)$ or $U_{L}(N) \times U_{R}(N)$, in this problem? It is known that in massless QED, without external fields, the chiral symmetry is $U_{L}(N) \times U_{R}(N)$ : though the singlet axial current $j_{5}^{\mu}$ is not conserved, the corresponding charge $Q_{5}$ is conserved. The latter is connected with the absence of instanton-like configurations in QED. In the presence of an external magnetic field, the situation however might be different. Indeed, the dynamics in QED in a magnetic field is intimately connected with the Schwinger model where the $U_{A}(1)$ symmetry is explicitly broken. We believe that this issue deserves further study.

An important ingredient of the dynamics in QED in a magnetic field is the pseudo-Higgs effect. It is not a genuine Higgs effect since there is no complete screening of the electric charge: Eq. (18) implies that in the deep infrared region with $\left|q_{\|}^{2}\right| \ll m_{d y n}^{2}$, there are ordinary Coulomb-like forces. Still, the pseudo-Higgs effect is manifested in creating a resonance in the photon channel, with $M_{\gamma}^{2}=2 \bar{\alpha}|e B| / \pi \gg m_{d y n}^{2}$. This resonance provides the dominating forces leading to chiral symmetry breaking.

Thus, in this problem, the region primarily responsible for chiral symmetry breaking is the region of intermediate momenta: $m_{d y n}^{2} \ll\left|q_{\|}^{2}\right| \ll|e B|$ and $m_{d y n}^{2} \ll M_{\gamma}^{2} \lesssim\left|q_{\perp}^{2}\right| \ll|e B|$. This point is noticeable as an example for a possibility discussed for the QCD dynamics [26]: the dynamics of spontaneous chiral symmetry breaking might be provided by forces essentially independent of the dynamics of confinement (the infrared dynamics).

Another noticeable point is the existence of a special gauge in which the description of the nonperturbative dynamics is essentially simplified. Recall that the conventional viewpoint now is that there is a particular gauge in QCD (the maximal Abelian gauge) which is the most appropriate one for the description of confinement and chiral symmetry breaking 29 .

At last, there are arguments in support of a dimensional reduction in the dynamics of chiral symmetry breaking in QCD [33]. The present model yields an example of such a mechanism.

We hope that the dynamics of the magnetic catalysis of chiral symmetry breaking in QED will provide insight into the non-perturbative dynamics of more complicated theories, such as quantum chromodynamics.

\section{ACKNOWLEDGMENTS}

We are grateful to Anthony Hams for his generous help in numerical solving the Schwinger-Dyson equations. We thank H. Minakata, A. Smilga, L.C.R. Wijewardhana and K. Yamawaki for useful discussions. The work of V.P.G. was supported by Swiss National Science Foundation Grant No. CEEC/NIS/96-98/7 IP 051219 and Foundation of Fundamental Researches of Ministry of Science of Ukraine under Grant No.2.5.1/003. The work of I.A.S. was supported by U.S. Department of Energy Grant \#DE-FG02-84ER40153. 


\section{APPENDIX A: LOOP CORRECTIONS TO THE VERTEX}

In this Appendix we will calculate the one and two-loop corrections to the vertex in the gauge (26) (see Fig.5). In agreement with the general arguments in Sec. III, it will be shown that they are small: of order $\alpha$ and $\alpha^{2}$ in one-loop and two-loop approximations, respectively. On the other hand, we will show that in covariant gauges (17), there is a large, $O(1)$, correction to the vertex already in the one-loop approximation.

As was pointed out in Sec. III, the loop expansion we use is based on the effective action of Cornwall, Jackiw, and Tomboulis. In this expansion, the full fermion and photon propagators are used and only two-particle irreducible graphs have to be taken into account 25,26. Our aim is to show that in the gauge (26), the improved rainbow approximation is reliable, i.e., the loop corrections to the vertex, with the photon and fermion propagators calculated in that approximation, are small. In this connection, notice that in the improved rainbow approximation the function $A\left(p_{\|}^{2}\right)$ in the fermion propagator equals one [see Eq. (41)] and $B\left(p_{\|}^{2}\right) \simeq m_{d y n}$ for all $p_{\|}^{2} \lesssim 2|e B|$ (see Fig. 3 ). Therefore one can use the bare propagator (16) with $m=m_{d y n}$ in the calculations of the loop corrections to the vertex: indeed, as was indicated in Sec. III, the perturbative expansion in $\alpha$ potentially might be destroyed by mass singularities coming from infrared region, where $B\left(p_{\|}^{2}\right) \simeq m_{d y n}$.

After getting rid of the Schwinger phase factors according to the prescription in Eq. (14), we get the following expression for the one-loop correction to the vertex function,

$$
\tilde{\Gamma}^{(1) \mu}(x-z, y-z)=(i e)^{2} \exp \left(i(x-y)^{\lambda} A_{\lambda}(z-y)\right) \gamma^{\lambda} \tilde{S}^{(L)}(x-z) \gamma^{\mu} \tilde{S}^{(L)}(z-y) \gamma^{\nu} \mathcal{D}_{\lambda \nu}(x-y)
$$

(see Fig. 5a). By performing the Fourier transform in both $x-z$ and $y-z$, we arrive at

$$
\begin{aligned}
\tilde{\Gamma}^{(1) \mu}(p, k) & =(i e)^{2} \int d^{4} x d^{4} y \frac{d^{4} k_{1} d^{4} k_{2} d^{4} q}{(2 \pi)^{12}} e^{i x p-i y k-i x A(y)-i k_{1} x+i k_{2} y+i q(x-y)} \gamma^{\lambda} \tilde{S}^{(L)}\left(k_{1}\right) \gamma^{\mu} \tilde{S}^{(L)}\left(k_{2}\right) \gamma^{\nu} \mathcal{D}_{\lambda \nu}(q) \\
& =(i e)^{2} \int d^{4} x \frac{d^{4} k_{1} d^{4} q}{(2 \pi)^{8}} e^{-i x k_{1}} \gamma^{\lambda} \tilde{S}^{(L)}\left(k_{1}+q+p\right) \gamma^{\mu} \tilde{S}^{(L)}(q+k-\vec{A}(x)) \gamma^{\nu} \mathcal{D}_{\lambda \nu}(q)
\end{aligned}
$$

where we first integrated over $y$ and $k_{2}$, and then shifted $k_{1}$ by $p+q$.

Substituting the fermion propagator as in Eq. (16), but with $m=m_{d y n}$, into the last expression, we obtain

$$
\begin{aligned}
\tilde{\Gamma}^{(1) \mu}(p, k) & =\frac{\alpha}{2 \pi^{3}} \int \frac{d^{2} q_{\|} d^{2} q_{\perp} \exp \left[-\left(q_{\perp}+p_{\perp}\right)^{2} l^{2} / 2-\left(q_{\perp}+k_{\perp}\right)^{2} l^{2} / 2+i l^{2}\left[q_{\perp} \times\left(p_{\perp}-k_{\perp}\right)\right]+i l^{2}\left[p_{\perp} \times k_{\perp}\right]\right]}{\left[\left(q_{\|}+p_{\|}\right)^{2}-m_{d y n}^{2}\right]\left[\left(q_{\|}+k_{\|}\right)^{2}-m_{d y n}^{2}\right]} \\
& \times \gamma^{\lambda}\left(\hat{q}_{\|}+\hat{p}_{\|}+m_{d y n}\right) O^{(-)} \gamma_{\|}^{\mu}\left(\hat{q}_{\|}+\hat{k}_{\|}+m_{d y n}\right) O^{(-)} \gamma^{\nu} \mathcal{D}_{\lambda \nu}\left(q_{\|}, q_{\perp}\right),
\end{aligned}
$$

where $\left[q^{\perp} \times p^{\perp}\right] \equiv \epsilon_{3 i j} q_{i} p_{j}$ (with $i, j=1,2$ ). As is clear from this expression, the dependence on the perpendicular momenta is weak in infrared, $0<\left|p_{\perp}\right|,\left|k_{\perp}\right| \lesssim 1 / l$, and exponentially suppressed in ultraviolet. For our purposes, it is sufficient to use $\left|p_{\perp}\right|,\left|k_{\perp}\right|=0$. Regarding the parallel components, we keep them non-zero so far.

After substituting the photon propagator (26) into Eq. (A3), we notice that the result contains two kinds of terms, namely, terms proportional to $O^{(+)}=\left(1+i \gamma^{1} \gamma^{2} \operatorname{sign}(e B)\right) / 2$ and terms proportional to $O^{(-)}=\left(1-i \gamma^{1} \gamma^{2} \operatorname{sign}\right)(e B) / 2$. As we discuss in Sec. IV, the former are completely irrelevant for the SD equation and we drop them. The latter are relevant and has to be carefully analyzed. As is easy to check, they come exclusively from the $g_{\mu \nu}^{\|}$term in the photon propagator, and the explicit expression (in Euclidean space) reads

$$
\tilde{\Gamma}^{(1) \mu}\left(p_{\|}, k_{\|}\right) \simeq \frac{\alpha m_{d y n}}{\pi^{3}} \int \frac{d^{2} q_{\|} d^{2} q_{\perp} e^{-\left(q_{\perp} l\right)^{2}}\left[\gamma_{\|}^{\mu}\left(\hat{q}_{\|}+\hat{p}_{\|}\right)+\left(\hat{q}_{\|}+\hat{k}_{\|}\right) \gamma_{\|}^{\mu}\right] O^{(-)}}{\left[\left(q_{\|}+p_{\|}\right)^{2}+m_{d y n}^{2}\right]\left[\left(q_{\|}+k_{\|}\right)^{2}+m_{d y n}^{2}\right]\left(q_{\|}^{2}+q_{\perp}^{2}+M_{\gamma}^{2}\right)} .
$$

In a standard way, we introduce the Feynman parameters and perform the integration over $q_{\|}$. The result reads,

$$
\begin{aligned}
\tilde{\Gamma}^{(1) \mu}\left(p_{\|}, k_{\|}\right) & \simeq \frac{\alpha}{\pi} m_{d y n} O^{(-)} \\
\times & \int_{0}^{\infty} d z e^{-z l^{2}} \int_{0}^{1} d x \int_{0}^{1-x} d y \frac{(1-y) \hat{k}_{\|} \gamma_{\|}^{\mu}-y \gamma_{\|}^{\mu} \hat{k}_{\|}-x \hat{p}_{\|} \gamma_{\|}^{\mu}+(1-x) \gamma_{\|}^{\mu} \hat{p}_{\|}}{\left[x\left(p_{\|}^{2}+m_{d y n}^{2}\right)+y\left(k_{\|}^{2}+m_{d y n}^{2}\right)-\left(x p_{\|}+y k_{\|}\right)^{2}+(1-x-y)\left(z+M_{\gamma}^{2}\right)\right]^{2}}
\end{aligned}
$$


Note that this is zero for $k_{\|}=p_{\|}=0$, and that the integral in the right hand side is finite for any finite $m_{d y n}^{2}$. In the most interesting region of momenta, $m^{2} \ll p_{\|}^{2}, k_{\|}^{2} \ll 1 / l^{2}$, this integral contains the logarithmic contribution, $\ln \left(m_{d y n} l\right)^{2}$.

While estimating the effect of the one-loop correction in Eq. (A5) to the SD equation in Sec. IV], we could check that the most important contribution comes from the region of momenta (up to the exchange of $p_{\|}$and $k_{\|}$) $m^{2} \ll p_{\|}^{2} \ll 1 / l^{2}$ and $k_{\|} \simeq 0$. In this particular case, from Eq. (A5) we obtain

$$
\begin{aligned}
& \tilde{\Gamma}^{(1) \mu}\left(p_{\|}, 0\right) \simeq \frac{\alpha}{\pi} m_{d y n} O^{(-)} \int_{0}^{\infty} \frac{d z e^{-z l^{2}}}{z+M_{\gamma}^{2}} \\
\times & \left(\frac{\gamma_{\|}^{\mu} \hat{p}_{\|}}{p_{\|}^{2}} \ln \frac{\left(p_{\|}^{2}\right)^{2}}{m_{d y n}^{2}\left(z+M_{\gamma}^{2}+p_{\|}^{2}\right)}-\frac{\hat{p}_{\|} \gamma_{\|}^{\mu}}{p_{\|}^{2}} \ln \frac{p_{\|}^{2}\left(z+M_{\gamma}^{2}+p_{\|}^{2}\right)}{m_{d y n}^{2}\left(z+M_{\gamma}^{2}\right)}+\frac{\hat{p}_{\|} \gamma_{\|}^{\mu}}{z+M_{\gamma}^{2}+p_{\|}^{2}} \ln \frac{\left(z+M_{\gamma}^{2}+p_{\|}^{2}\right)^{2}}{m_{d y n}^{2}\left(z+M_{\gamma}^{2}\right)}\right) \\
\simeq & \frac{\alpha}{\pi} m_{d y n} O^{(-)}\left(\frac{\gamma_{\|}^{\mu} \hat{p}_{\|}}{p_{\|}^{2}} \ln \frac{p_{\|}^{2}}{m_{d y n}^{2}} \ln \frac{1}{\left(M_{\gamma} l\right)^{2}}-\frac{\hat{p}_{\|} \gamma_{\|}^{\mu}}{p_{\|}^{2}} \ln \frac{1}{\left(m_{d y n} l\right)^{2}} \ln \frac{1}{\left(M_{\gamma} l\right)^{2}+\left(p_{\|} l\right)^{2}}\right) .
\end{aligned}
$$

This, in its turn, results in a suppressed two-loop correction to the SD equation.

Now, let us consider the correction to vertex function at two-loop order. There are two diagrams in this order (see Fig. 51. Here we shall explicitly describe the correction connected with the diagram with two crossed photon lines (Fig. 5b); the analysis of another correction, connected with the diagram with two parallel photon lines (Fig. 5ic) can be done similarly.

In coordinate space, the expression corresponding to the diagram with two crossed photon lines reads

$$
\begin{aligned}
\tilde{\Gamma}^{(2) \mu}(x, y) & =(i e)^{4} \int d^{4} x_{1} d^{4} y_{1} \exp \left[i x^{\lambda} A_{\lambda}\left(x_{1}\right)+i y_{1}^{\lambda} A_{\lambda}(y)+i y^{\lambda} A_{\lambda}(x)\right] \gamma^{\sigma} \tilde{S}^{(L)}\left(x-x_{1}\right) \gamma^{\nu} \tilde{S}^{(L)}\left(x_{1}\right) \\
& \times \gamma^{\mu} \tilde{S}^{(L)}\left(-y_{1}\right) \gamma^{\rho} \tilde{S}^{(L)}\left(y_{1}-y\right) \gamma^{\lambda} \mathcal{D}_{\nu \lambda}\left(x_{1}-y\right) \mathcal{D}_{\sigma \rho}\left(x-y_{1}\right) .
\end{aligned}
$$

In the momentum space, this becomes

$$
\begin{aligned}
\tilde{\Gamma}^{(2) \mu}(p, k) & =(i e)^{4} \int d^{4} x d^{4} y d^{4} x_{1} d^{4} y_{1} \frac{d^{4} k_{1} d^{4} k_{2} d^{4} k_{3} d^{4} k_{4} d^{4} q_{1} d^{4} q_{2}}{(2 \pi)^{24}} \\
& \times e^{i x p-i y k+i x A\left(x_{1}\right)+i y_{1} A(y)-i x A(y)-i k_{1}\left(x-x_{1}\right)-i k_{2} x_{1}+i k_{3} y_{1}-i k_{4}\left(y_{1}-y\right)+i q_{1}\left(x_{1}-y\right)+i q_{2}\left(x-y_{1}\right)} \\
& \times \gamma^{\sigma} \tilde{S}^{(L)}\left(k_{1}\right) \gamma^{\nu} \tilde{S}^{(L)}\left(k_{2}\right) \gamma^{\mu} \tilde{S}^{(L)}\left(k_{3}\right) \gamma^{\rho} \tilde{S}^{(L)}\left(k_{4}\right) \gamma^{\lambda} \mathcal{D}_{\nu \lambda}\left(q_{1}\right) \mathcal{D}_{\sigma \rho}\left(q_{2}\right) .
\end{aligned}
$$

Performing the straightforward integrations over $x_{1}$ and $y_{1}$, and then over $k_{i}$ (by making use of the explicit form of the fermion propagator), at the end we arrive at the following expression for the two-loop vertex correction at $k=p=0$ (the case $k, p \neq 0$ is not expected to give a very different estimate, but it is much harder to work with),

$$
\begin{aligned}
\tilde{\Gamma}^{(2) \mu}(0,0)= & \frac{\alpha^{2}}{\pi^{6}} \int \frac{d^{2} q_{1}^{\|} d^{2} q_{2}^{\|} d^{2} q_{1}^{\perp} d^{2} q_{2}^{\perp} \exp \left[-\frac{3}{2}\left(q_{1}^{\perp} l\right)^{2}-\frac{3}{2}\left(q_{2}^{\perp} l\right)^{2}+2 i l^{2}\left[q_{1}^{\perp} \times q_{2}^{\perp}\right]-2 l^{2} q_{1}^{\perp} \cdot q_{2}^{\perp}\right]}{\left(\left(q_{1}^{\|}\right)^{2}-m_{d y n}^{2}\right)\left(\left(q_{2}^{\|}\right)^{2}-m_{d y n}^{2}\right)\left[\left(q_{1}^{\|}+q_{2}^{\|}\right)^{2}-m_{d y n}^{2}\right]^{2}} \\
& \times \gamma^{\sigma}\left(\hat{q}_{2}^{\|}+m_{d y n}\right) O^{(-)} \gamma^{\nu}\left(\hat{q}_{1}^{\|}+\hat{q}_{2}^{\|}+m_{d y n}\right) O^{(-)} \gamma^{\mu}\left(\hat{q}_{1}^{\|}+\hat{q}_{2}^{\|}+m_{d y n}\right) O^{(-)} \\
& \times \gamma^{\rho}\left(\hat{q}_{1}^{\|}+m_{d y n}\right) O^{(-)} \gamma^{\lambda} \mathcal{D}_{\nu \lambda}\left(q_{1}^{\|}, q_{1}^{\perp}\right) \mathcal{D}_{\sigma \rho}\left(q_{2}^{\|}, q_{2}^{\perp}\right) .
\end{aligned}
$$

Switching to the Euclidean space and substituting the photon propagator in the Feynman like gauge (26), we find that the only nonzero contribution is proportional to $O^{(-)}$,

$$
\begin{aligned}
& \tilde{\Gamma}^{(2) \mu}(0,0)=\frac{4 \alpha^{2} m_{d y n}^{4}}{\pi^{6}} \gamma_{\|}^{\mu} O^{(-)} \\
& \times \int \frac{d^{2} q_{1} d^{2} q_{2} d^{2} q_{1}^{\perp} d^{2} q_{2}^{\perp} \exp \left[-\frac{3}{2}\left(q_{1}^{\perp} l\right)^{2}-\frac{3}{2}\left(q_{2}^{\perp} l\right)^{2}+2 i l^{2}\left[q_{1}^{\perp} \times q_{2}^{\perp}\right]-2 l^{2} q_{1}^{\perp} \cdot q_{2}^{\perp}\right]}{\left(q_{1}^{2}+m_{d y n}^{2}\right)\left(q_{2}^{2}+m_{d y n}^{2}\right)\left[\left(q_{1}+q_{2}\right)^{2}+m_{d y n}^{2}\right]^{2}\left(q_{1}^{2}+\left(q_{1}^{\perp}\right)^{2}+M_{\gamma}^{2}\right)\left(q_{2}^{2}+\left(q_{2}^{\perp}\right)^{2}+M_{\gamma}^{2}\right)} \\
& \leq \text { Const } \gamma_{\|}^{\mu} O^{(-)} \alpha^{2} \ln ^{2}\left(\frac{1+\left(M_{\gamma} l\right)^{2}}{\left(M_{\gamma} l\right)^{2}}\right) .
\end{aligned}
$$


If we assume that $N$ is not too large, so that $\left(M_{\gamma} l\right)^{2} \simeq 2 \bar{\alpha} / \pi \ll 1$, then the above estimate for the two-loop correction becomes

$$
\tilde{\Gamma}^{(2) \mu}(0,0) \leq \text { Const } \gamma_{\|}^{\mu} O^{(-)} \alpha^{2} \ln ^{2}\left(\frac{\pi}{2 \bar{\alpha}}\right) .
$$

Thus, we conclude that, as expected, the Feynman like non-local gauge leads to suppressed higher order corrections. The latter, in its turn, means that the solution to the SD equation with the bare vertex and the photon propagator in Eq. (26) is reliable, and that the result presumably approaches the exact one when the fine structure constant is very small.

At this point it is instructive to explicitly demonstrate that the observed suppression in higher orders is the exclusive property of the special gauge in Eq. (26).

To prove this, let us consider the one-loop vertex correction in the case of the covariant gauge (17). After substituting the photon propagator into Eq. (A3), we arrive at the following estimate

$$
\begin{aligned}
\tilde{\Gamma}^{(1) \mu}(0,0) \simeq & \frac{\alpha}{4 \pi} \gamma_{\|}^{\mu} O^{(-)} \ln ^{2}\left(\frac{1}{\left(m_{\text {dyn }} l\right)^{2}}\right)-\frac{\alpha}{4 \pi} \gamma_{\|}^{\mu} O^{(-)} \ln \left(\frac{M_{\gamma}^{2}}{m_{d y n}^{2}\left(m_{\text {dyn }} l\right)^{2}}\right) \ln \left(\frac{1}{\left(M_{\gamma} l\right)^{2}}\right) \\
& -\lambda \frac{\alpha}{2 \pi} \gamma_{\|}^{\mu} O^{(-)} \ln \left(\frac{1}{\left(m_{\text {dyn }} l\right)^{2}}\right),
\end{aligned}
$$

where, as is easy to check, the leading term with double logarithm comes from the $q_{\mu}^{\|} q_{\nu}^{\|} / q^{2} q_{\|}^{2}$ component in the photon propagator in Eq. (17). Since in the rainbow approximation the solution to the SD equation in the covariant gauge yields $\ln ^{2}\left(m_{d y n} l\right)^{2} \sim 1 / \alpha$ [see Eq. (11)], we conclude that the one-loop correction in Eq. (A12) is of the same order as the bare vertex, i.e., $\tilde{\Gamma}^{(1) \mu}(0,0)=O(1)$. As a result, such an approximation is not self-consistent.

Notice that if $M_{\gamma}^{2}$ were equal to zero (i.e., $\Pi\left(q_{\perp}^{2}, q_{\|}^{2}\right)=0$ in Eq. (17) and the $q_{\mu}^{\|} q_{\nu}^{\|} / q^{2} q_{\|}^{2}$ term would be absent there), the first two terms in Eq. (A12) would cancel, and the one-loop correction $\tilde{\Gamma}^{(1) \mu}(0,0)$ would be small $\left(\tilde{\Gamma}^{(1) \mu}(0,0) \sim\right.$ $O(\sqrt{\alpha})$ ). As a result, expression (11) for $m_{d y n}$ would be correct. Therefore the origin of the deviation of expressions (1) and (2) for $m_{d y n}$ is the generation of a non-zero $M_{\gamma}$.

We emphasize that the $q_{\mu}^{\|} q_{\nu}^{\|} / q^{2} q_{\|}^{2}$ component in the photon propagator, responsible for breaking the consistency of the rainbow approximation to the SD equation, is absent in the gauge Eq. (26). As a result, in this gauge, there exists a consistent truncation of the SD equation for small values of the coupling constant. Since the dynamical mass is a gauge invariant quantity, in other gauges one needs to sum up an infinite set of diagrams to recover the same result. Obviously it is rather difficult to classify all the relevant diagrams in those gauges, and so the existence of the special gauge is the key point that allows to solve the problem of the magnetic catalysis in QED reliably.

\section{APPENDIX B: ANALYTICAL ANALYSIS OF THE SD EQUATION}

In this appendix we will describe the analytical solution of the SD equation (47).

First of all, let us show that the leading singularity, $1 / \alpha \log \alpha$, in $\log \left(m_{d y n}^{2}\right)$ in Eq. (49) is induced in the kinematic region with $m_{\mathrm{dyn}}^{2} \ll\left|q_{\|}^{2}\right| \ll|e B|$ and $m_{d y n}^{2} \ll M_{\gamma}^{2} \lesssim q_{\perp}^{2} \ll|e B|$ (in that region, fermions can be treated as massless).

As was shown in Sec. $\mathrm{V}$, the approximation with $B\left(p_{\|}^{2}\right)=m_{\mathrm{dyn}}$ for $p_{\|}^{2}<2|e B|$ and $B\left(p_{\|}^{2}\right)$ rapidly decreasing for $p_{\|}^{2}>2|e B|$ is reliable in this problem (see Fig. 3). Then, taking $p_{\|}^{2}=0$ in Eq. (47), we arrive at the equation

$$
\begin{aligned}
1 & =\frac{\alpha}{2 \pi^{2}} \int^{2|e B|} \frac{d^{2} q_{\|}}{q_{\|}^{2}+m_{d y n}^{2}} \int_{0}^{\infty} \frac{d x \exp \left(-x l^{2} / 2\right)}{x+q_{\|}^{2}+q_{\|}^{2} \Pi_{E}\left(x, q_{\|}^{2}\right)} \\
& \simeq \frac{\alpha}{2 \pi^{2}} \int^{2|e B|} \frac{d^{2} q_{\|}}{q_{\|}^{2}+m_{d y n}^{2}} \int_{0}^{2|e B|} \frac{d x}{x+q_{\|}^{2}+q_{\|}^{2} \Pi_{E}\left(x, q_{\|}^{2}\right)} .
\end{aligned}
$$

Matching now the asymptotes (18),(19) at $q_{\|}^{2}=6 m_{d y n}$ in Euclidean space, we get 


$$
\begin{aligned}
1 & \simeq \frac{\alpha}{2 \pi} \int_{0}^{2|e B|} d x\left[\int_{0}^{6 m_{d y n}^{2}} \frac{d y}{\left(y+m_{d y n}^{2}\right)\left(x+y\left(1+\frac{M_{\gamma}^{2}}{6 m_{\mathrm{dyn}}^{2}}\right)\right)}\right. \\
& \left.+\int_{6 m_{\mathrm{dyn}}^{2}}^{2|e B|} \frac{d y}{\left(y+m_{d y n}^{2}\right)\left(x+y+M_{\gamma}^{2} e^{-x l^{2} / 2}\right)}\right] .
\end{aligned}
$$

It is clear that, because of $m_{d y n}^{2}$ in $\left(y+m_{d y n}^{2}\right)$, the first term in the square bracket on the right hand side of this equation is of order $O(1)$ and can be neglected: it cannot give a contribution of order $1 / \alpha \log \alpha$ to $m_{d y n}^{2}$. Then we arrive at the estimate,

$$
1 \simeq \frac{\alpha}{2 \pi} \int_{6 m_{\mathrm{dyn}}^{2}}^{2|e B|} \frac{d y}{y+m_{d y n}^{2}} \int_{0}^{2|e B|} \frac{d x}{x+y+M_{\gamma}^{2} e^{-x l^{2} / 2}}
$$

The double logarithmic contribution comes from the region $2|e B| \gg y=q_{\|}^{2} \gg m_{d y n}^{2}, 2|e B| \gg x=q_{\perp}^{2} \gtrsim y+M_{\gamma}^{2} \geq$ $M_{\gamma}^{2}, M_{\gamma}^{2}=2 \bar{\alpha} / \pi l^{2}$. Therefore one can write

$$
1 \simeq \frac{\alpha}{2 \pi} \int_{6 m_{d y n}^{2}}^{2|e B|} \frac{d y}{y} \int_{y+M_{\gamma}^{2}}^{2|e B|} \frac{d x}{x}=\frac{\alpha}{2 \pi} \int_{6 m_{d y n}^{2}}^{2|e B|} \frac{d y}{y} \log \frac{2|e B|}{y+M_{\gamma}^{2}} .
$$

To calculate the last integral with double logarithmic accuracy, we write

$$
\begin{aligned}
1 & \simeq \frac{\alpha}{2 \pi}\left[\log \frac{2 e B}{M_{\gamma}^{2}} \int_{6 m_{d y n}^{2}}^{M_{\gamma}^{2}} \frac{d y}{y}+\int_{M_{\gamma}^{2}}^{2|e B|} \frac{d y}{y} \log \frac{2|e B|}{y}\right] \\
& \simeq \frac{\alpha}{2 \pi}\left[\log \frac{2 e B}{M_{\gamma}^{2}} \log \frac{M_{\gamma}^{2}}{m_{d y n}^{2}}+\frac{1}{2} \log ^{2} \frac{2 e B}{M_{\gamma}^{2}}\right]=\frac{\alpha}{4 \pi} \log \frac{2 e B}{M_{\gamma}^{2}} \log \left[\frac{2|e B| M_{\gamma}^{2}}{m_{d y n}^{4}}\right] .
\end{aligned}
$$

This equation implies that

$$
m_{d y n} \sim \sqrt{|e B|}\left(\frac{N \alpha}{\pi}\right)^{1 / 4} \exp \left(-\frac{\pi}{\alpha \log \frac{\pi}{N \alpha}}\right) .
$$

Comparing this expression with Eq. (49), one can see that this estimate is quite reasonable. The origin of that is a rather simple form of the fermion mass function $B\left(p_{\|}^{2}\right): B\left(p_{\|}^{2}\right) \simeq m_{d y n}$ for $p_{\|}^{2} \lesssim 2|e B|$ and $B\left(p_{\|}^{2}\right)$ rapidly decreases for $p_{\|}^{2} \gtrsim 2|e B|$.

Therefore the dominant contribution to the SD equation (47) comes from the region with $m_{\text {dyn }}^{2} \ll\left|q_{\|}^{2}\right| \ll|e B|$ and $m_{d y n}^{2} \ll M_{\gamma}^{2} \lesssim q_{\perp}^{2} \ll|e B|$, where fermions can be treated as massless. This in turn justifies the approximation with the polarization function $\Pi_{E}=2 \bar{\alpha}|e B| / \pi q_{\|}^{2}$.

Now we proceed at solving analytically SD equation (47) for a mass function in the improved ladder approximation. It is

$$
B(p)=\frac{\alpha}{2 \pi^{2}} \int \frac{d^{2} k B(k)}{k^{2}+B^{2}(k)} \int_{0}^{\infty} \frac{d z e^{-z l^{2} / 2}}{z+(k-p)^{2}+\Pi(z)}, \quad \Pi(z)=M_{\gamma}^{2} e^{-z l^{2} / 2},
$$

where $M_{\gamma}^{2}=2 \bar{\alpha}|e B| / \pi$ and we shifted the momentum of integration (also we omit the symbol $\|$ in the rest of this appendix). After the integration over the angular coordinate, Eq. (B7) becomes

$$
B\left(p^{2}\right)=\frac{\alpha}{2 \pi} \int \frac{d k^{2} B\left(k^{2}\right)}{k^{2}+B^{2}\left(k^{2}\right)} K\left(p^{2}, k^{2}\right)
$$


with the kernel

$$
K\left(p^{2}, k^{2}\right)=\int_{0}^{\infty} \frac{d z \exp \left(-z l^{2} / 2\right)}{\sqrt{\left(p^{2}+k^{2}+M_{\gamma}^{2} e^{-z l^{2} / 2}+z\right)-4 p^{2} k^{2}}} .
$$

To study Eq. (B8) analytically, we break up the momentum integration into two regions and expand the kernel appropriately for each region (compare with Refs. 34, 33)

$$
\begin{aligned}
B\left(p^{2}\right) & =\frac{\alpha}{2 \pi}\left[\int_{0}^{p^{2}} \frac{d k^{2} B\left(k^{2}\right)}{k^{2}+B^{2}\left(k^{2}\right)} \int_{0}^{\infty} \frac{d z \exp \left(-z l^{2} / 2\right)}{p^{2}+M_{\gamma}^{2} e^{-z l^{2} / 2}+z}\right. \\
& \left.+\int_{p^{2}}^{\infty} \frac{d k^{2} B\left(k^{2}\right)}{k^{2}+B^{2}\left(k^{2}\right)} \int_{0}^{\infty} \frac{d z \exp \left(-z l^{2} / 2\right)}{k^{2}+M_{\gamma}^{2} e^{-z l^{2} / 2}+z}\right] .
\end{aligned}
$$

Introducing dimensionless variables $x=p^{2} l^{2} / 2, y=k^{2} l^{2} / 2$ and also the dimensionless mass function $B\left(p^{2}\right) / \sqrt{2|e B|} \rightarrow$ $B(x)$, we rewrite the last equation in the form

$$
B(x)=\frac{\alpha}{2 \pi}\left[g(x) \int_{0}^{x} \frac{d y B(y)}{y+B^{2}(y)}+\int_{x}^{\infty} \frac{d y B(y) g(y)}{y+B^{2}(y)}\right],
$$

where

$$
g(x)=\int_{0}^{\infty} \frac{d z e^{-z}}{z+x+\frac{\bar{\alpha}}{\pi} e^{-z}} .
$$

The solutions of the integral equation (B11) satisfy the second-order differential equation

$$
B^{\prime \prime}-\frac{g^{\prime \prime}}{g^{\prime}} B^{\prime}-\frac{\alpha}{2 \pi} g^{\prime} \frac{B}{x+B^{2}(x)}=0,
$$

where the prime denotes derivative with respect to $x$. The boundary conditions are

$$
\begin{gathered}
\left.\frac{B^{\prime}}{g^{\prime}}\right|_{x=0}=0, \\
\left.\left(B-\frac{g B^{\prime}}{g^{\prime}}\right)\right|_{x=\infty}=0 .
\end{gathered}
$$

The function $g(x)$ has asymptotic behavior

$$
\begin{aligned}
& g(x) \sim \log \frac{1+\frac{\bar{\alpha}}{\pi}}{x+\frac{\bar{\alpha}}{\pi}}, \quad x \ll 1, \\
& g(x) \sim \frac{1}{x}, \quad x \gg 1 .
\end{aligned}
$$

We consider now the linearized version of Eq. (B13) when the term $B^{2}(x)$ in denominator is replaced by a constant $B^{2}(0) \equiv a^{2}\left(B(p=0) \equiv m_{d y n}\right)$ : the numerical analysis shows that it is an excellent approximation. The two independent solutions of that equation near the point $x=\infty$ behave as $B(x) \sim$ const and $B(x) \sim 1 / x$, and the UVBC selects the last one.

In the region $x \ll 1$, the equation takes the form

$$
B^{\prime \prime}+\frac{1}{x+\frac{\bar{\alpha}}{\pi}} B^{\prime}+\frac{\alpha}{2 \pi} \frac{B}{\left(x+\frac{\bar{\alpha}}{\pi}\right)\left(x+a^{2}\right)}=0 .
$$


Introducing the variable $x+a^{2}=-z\left(\bar{\alpha} / \pi-a^{2}\right)$, Eq. (B17) can be rewritten in the form of an equation for the hypergeometric function,

$$
z(1-z) \frac{d^{2} B}{d z^{2}}-z \frac{d B}{d z}-\frac{\alpha}{2 \pi} B=0 .
$$

The general solution to Eq. (B18) has the form

$$
B(z)=C_{1} u_{1}+C_{2} u_{2}
$$

where

$$
\begin{gathered}
u_{1}=z F(1+i \nu, 1-i \nu ; 2 ; z), \\
u_{2}=(-z)^{-i \nu} F\left(i \nu, 1+i \nu ; 1+2 i \nu ; \frac{1}{z}\right)+(-z)^{i \nu} F\left(-i \nu, 1-i \nu ; 1-2 i \nu ; \frac{1}{z}\right),
\end{gathered}
$$

$\nu=\sqrt{\alpha / 2 \pi}$. From the infrared boundary condition (B14), one gets

$$
\frac{C_{2}}{C_{1}}=-\left.\frac{u_{1}^{\prime}}{u_{2}^{\prime}}\right|_{x=0}
$$

Equating now the logarithmic derivatives of solution (B19) (at $x \ll 1)$ and $1 / x$ (at $x \gg 1$ ) at the point $x=1$, we arrive at the equation determining the quantity $a(\alpha)$ (i.e., the dynamical mass $\left.m_{d y n}\right)$ :

$$
\varphi \equiv A_{1} B_{2}-A_{2} B_{1}=0
$$

where

$$
A_{i}=\left.\left(u_{i}^{\prime}+u_{i}\right)\right|_{x=1}, \quad B_{i}=\left.u_{i}^{\prime}\right|_{x=0}
$$

Since the variable $z$ is

$$
z=-\left.\frac{x+a^{2}}{\frac{\bar{\alpha}}{\pi}-a^{2}}\right|_{x=0} \simeq-\frac{\pi}{\bar{\alpha}} a^{2}, \quad z=-\left.\frac{x+a^{2}}{\frac{\bar{\alpha}}{\pi}-a^{2}}\right|_{x=1} \simeq-\frac{\pi}{\bar{\alpha}}
$$

(we suppose $a^{2} \ll \bar{\alpha} / \pi$ ), in what follows we need asymptotic behavior of $u_{i}(z), u_{i}^{\prime}(z)$ at small and large negative values of its argument $z$. Using corresponding formulas from [30], we find for small values of $z(|z| \ll 1)$

$$
\begin{aligned}
& u_{1} \simeq z\left(1+\frac{1+\nu^{2}}{2} z\right)+O\left(z^{2}\right) \\
& u_{2} \simeq 2 \operatorname{Re}\left\{\frac{\Gamma(1+2 i \nu)}{\Gamma^{2}(1+i \nu)}\left[\nu^{2} z\left(\ln (-z)-h_{0}\right)+1\right]+O\left(z^{2} \ln z\right)\right\}, \\
& u_{1}^{\prime} \simeq 1+\left(1+\nu^{2}\right) z+O\left(z^{2}\right), \\
& u_{2}^{\prime} \simeq 2 \operatorname{Re}\left\{\frac{\Gamma(1+2 i \nu)}{\Gamma^{2}(1+i \nu)} \nu^{2}\left(\ln (-z)+1-h_{0}\right)+O(z \ln z)\right\},
\end{aligned}
$$

where

$$
h_{0}=1-2 \gamma-\psi(i \nu)-\psi(1+i \nu)
$$

At large $|z| \gg 1$ we have

$$
\begin{aligned}
& u_{1} \simeq-\frac{1}{\nu} \sqrt{\frac{\tanh (\pi \nu)}{\pi \nu}} \sin [\nu \ln (-z)+\Phi(\nu)]+O\left(z^{-1}\right), \\
& u_{2} \simeq 2 \cos (\nu \ln (-z))+O\left(z^{-1}\right), \\
& u_{1}^{\prime} \simeq-\frac{1}{z} \sqrt{\frac{\tanh (\pi \nu)}{\pi \nu}} \cos (\nu \ln (-z)+\Phi(\nu))+O\left(z^{-2}\right), \\
& u_{2}^{\prime} \simeq-\frac{2 \nu}{z} \sin (\nu \ln (-z))+O\left(z^{-2}\right),
\end{aligned}
$$


where

$$
\begin{aligned}
\Phi(\nu) & =\arg \left(\frac{\Gamma(1+2 i \nu)}{\Gamma^{2}(1+i \nu)}\right)=\sum_{n=1}^{\infty}(-1)^{n+1} \frac{2\left(2^{2 n}-1\right) \zeta(2 n+1)}{2 n+1} \nu^{2 n+1} \\
& \simeq 2 \zeta(3) \nu^{3}-6 \zeta(5) \nu^{5}+\ldots
\end{aligned}
$$

By making use of these asymptotes, we obtain the following expressions for $A_{i}, B_{i}$,

$$
\begin{aligned}
& A_{1}=\left.\left(\frac{d u_{1}}{d x}+u_{1}\right)\right|_{x=1} \simeq-\sqrt{\frac{\tanh (\pi \nu)}{\pi \nu}}\left[\cos \left(\nu \ln \frac{\pi}{\bar{\alpha}}\right)+\frac{1}{\nu} \sin \left(\nu \ln \frac{\pi}{\bar{\alpha}}+\Phi(\nu)\right)\right], \\
& A_{2}=\left.\left(\frac{d u_{2}}{d x}+u_{2}\right)\right|_{x=1} \simeq 2 \cos \left(\nu \ln \frac{\pi}{\bar{\alpha}}\right)+2 \sin \left(\nu \ln \frac{\pi}{\bar{\alpha}}\right), \\
& B_{1}=\left.\frac{d u_{1}}{d x}\right|_{x=0} \simeq-\frac{\pi}{\bar{\alpha}} \\
& B_{2}=\left.\frac{d u_{2}}{d x}\right|_{x=0} \simeq-\ln \frac{\pi a^{2}}{\bar{\alpha}} .
\end{aligned}
$$

And, finally, the solution to Eq. (B23) reads

$$
a^{2}=\frac{m_{d y n}^{2}}{2|e B|} \simeq \frac{N \alpha}{\pi} \exp \left[-\frac{1}{\nu} \cot \left(\nu \ln \frac{\pi}{N \alpha}\right)\right] \simeq\left(\frac{N \alpha}{\pi}\right)^{2 / 3} \exp \left[-\frac{2 \pi}{\alpha \ln (\pi / N \alpha)}\right], \quad \text { as } \quad \alpha \rightarrow 0 .
$$

The obtained analytical expression (B41) for the dynamical mass is close both to estimate (B6) and to the numerical solution (49). The ratio of the values of $C_{1}$ in the analytical solution and in the numerical one is $C_{1}^{(\text {anal })} / C_{1}^{(\text {numer })} \simeq 1.7$. This, rather mild, discrepancy reflects the approximations made in the kernel of the integral equation (B8) in reducing it to the differential equation (B13).

\section{APPENDIX C: WARD-TAKAHASHI IDENTITY}

In this appendix, we discuss the simplest Ward-Takahashi (WT) identity relating the fermion propagator and the vertex in the problem of magnetic catalysis (for another approach see Ref. [13]).

Let us start from the WT identity in coordinate space:

$$
\partial_{\mu}^{z} \Gamma^{\mu}\left(x^{\prime}, y^{\prime} ; z\right)=\delta\left(x^{\prime}-z\right) G^{-1}\left(z, y^{\prime}\right)-\delta\left(z-y^{\prime}\right) G^{-1}\left(x^{\prime}, z\right),
$$

After multiplying this expression with $G\left(x, x^{\prime}\right)$ on the left and $G\left(y^{\prime}, y\right)$ on the right and integrating over $x^{\prime}$ and $y^{\prime}$, we arrive at another representation,

$$
\int d^{4} x^{\prime} d^{4} y^{\prime} G\left(x, x^{\prime}\right) \partial_{\mu}^{z} \Gamma^{\mu}\left(x^{\prime}, y^{\prime} ; z\right) G\left(y^{\prime}, y\right)=\delta(z-y) G(x, z)-\delta(x-z) G(z, y) .
$$

By taking into account the universal Schwinger phase factors of the propagators and the vertex [see Eq. (14) in Sec. II], this identity (in the momentum representation) reads

$$
i \tilde{G}\left(p_{2}\right)-i \tilde{G}\left(p_{1}\right)=\int d^{4} r \frac{d^{4} q}{(2 \pi)^{4}} e^{-i r q} \tilde{G}\left(p_{1}+A^{e x t}(r)\right)\left(p_{1}-p_{2}\right)_{\mu} \tilde{\Gamma}^{\mu}\left(p_{1}+q, p_{2}+q\right) \tilde{G}\left(p_{2}+q-A^{e x t}(r)\right) .
$$

Now, by substituting the bare vertex along with the solution for the fermion propagator as in Eq. (28) with $A\left(p_{\|}^{2}\right)=1$ and $B\left(p_{\|}^{2}\right)=m_{d y n}=$ Const (recall that the latter is a very good approximation in the most important region of momenta, $\left.p_{\perp, \|}^{2} \ll|e B|\right)$, we obtain the following relation

$$
\left[\left(\hat{p}_{1}^{\|}-m_{d y n}\right) e^{-\left(p_{2}^{\perp} l\right)^{2}}-\left(\hat{p}_{2}^{\|}-m_{d y n}\right) e^{-\left(p_{1}^{\perp} l\right)^{2}}\right] O^{(-)}=e^{-\left(p_{1}^{\perp} l\right)^{2} / 2-\left(p_{2}^{\perp} l\right)^{2} / 2+i l^{2}\left[p_{1}^{\perp} \times p_{2}^{\perp}\right]}\left(\hat{p}_{1}^{\|}-\hat{p}_{2}^{\|}\right) O^{(-)} .
$$

Once again, the approximation for the fermion propagator with $A\left(p_{\|}^{2}\right)=1$ and $B\left(p_{\|}^{2}\right)=m_{d y n}=$ Const is reliable only in the dynamical region $p_{\perp, \|}^{2} \ll|e B|$. Therefore, at best, one could expect that the WT identity is satisfied in 
this same region of momenta. After expanding both sides of Eq. ( $\mathrm{C} 4)$ in powers of $p_{\perp}^{2} l^{2}=p_{\perp}^{2} /|e B|$ and keeping only the leading order contribution, we see that Eq. (C4) indeed turns into an identity. In the end, we remind that the same conclusion about the WT identity was reached in Ref. [13] by making use of a different approach.

[1] V.P. Gusynin, V.A. Miransky, and I.A. Shovkovy, Phys. Rev. Lett. 73, 3499 (1994); Phys. Rev. D 52, 4718 (1995); Phys. Lett. B 349, 477 (1995).

[2] V.P. Gusynin, V.A. Miransky, and I.A. Shovkovy, Phys. Rev. D 52, 4747 (1995).

[3] V.P. Gusynin, V.A. Miransky, and I.A. Shovkovy, Nucl. Phys. B 462, 249 (1996).

[4] K. Farakos and N.E. Mavromatos, Int. J. Mod. Phys. B 12, 809 (1998); G.W. Semenoff, I.A. Shovkovy and L.C.R. Wijewardhana, Mod. Phys. Lett. A 13, 1143 (1998); W.V. Liu, cond-mat/9808134 (to appear in Nucl. Phys. B).

[5] V.A. Miransky, Prog. Theor. Phys. (Supplement) 123, 49 (1996).

[6] C.N. Leung, Y.J. Ng, and A.W. Ackley, Phys. Rev. D 54, 4181 (1996).

[7] D.-S. Lee, C.N. Leung, and Y.J. Ng, Phys. Rev. D 55, 6504 (1997); Phys. Rev. D 57, 5224 (1998).

[8] E.J. Ferrer and V. de la Incera, hep-ph/9810473.

[9] D.K. Hong, Y. Kim, and S.-J. Sin, Phys. Rev. D 54, 7879 (1996).

[10] V.P. Gusynin and I.A. Shovkovy, Phys. Rev. D 56, 5251 (1997).

[11] I.A. Shushpanov and A.V. Smilga, Phys. Lett. B 402, 351 (1997).

[12] D.K. Hong, Phys. Rev. D 57, 3759 (1998).

[13] E.J. Ferrer and V. de la Incera, Phys. Rev. D 58, 065008 (1998).

[14] V.P. Gusynin and A.V. Smilga, Phys. Lett. B 450, 267 (1999).

[15] D.-S. Lee, P.N. McGraw, Y.J. Ng and I.A. Shovkovy, Phys. Rev. D 59, 085008 (1999).

[16] V.P. Gusynin, V.A. Miransky, and I.A. Shovkovy, Phys. Rev. Lett. 83, 1291 (1999).

[17] A.I. Akhiezer and V.B. Berestetsky, "Quantum Electrodynamics" (Interscience, NY, 1965); D. M. Gitman, E.S. Fradkin and Sh.M. Shvartsman, in "Quantum Electrodynamics with Unstable Vacuum", edited by V.L. Ginzburg (Nova Science, Commack, NY, 1995).

[18] J. Schwinger, Phys. Rev. 82, 664 (1951).

[19] A. Chodos, I. Everding and D.A. Owen, Phys. Rev. D 42, 2881 (1990).

[20] V. Elias, D.G.C. Mckeon, V.A. Miransky, and I.A. Shovkovy, Phys. Rev. D 54, 7884 (1996).

[21] N.D. Mermin and H. Wagner, Phys. Rev. Lett. 17, 1133 (1966); S. Coleman, Commun. Math. Phys. 31, 259 (1973).

[22] Yu.M. Loskutov and V.V. Skobelev, Phys. Lett. A 56, 151 (1976); W. Dittrich and M. Reuter, "Effective Lagrangians in Quantum Electrodynamics" (Springer, Berlin, 1985); G. Calucci and R. Ragazzon, J. Phys. A 27, 2161 (1994).

[23] J. Schwinger, Phys. Rev. 125, 397 (1962); J.H. Lowenstein and J.A. Swieca, Ann. Phys. (NY) 68, 172 (1971).

[24] Y. Frishman, in "Particles, Quantum Fields and Statistical Mechanics", Lecture Notes in Physics, No. 32, edited by M. Alexanian and A. Zepeda (Springer, Berlin, 1975).

[25] J.M. Cornwall, R. Jackiw and E. Tomboulis, Phys. Rev. D 10, 2428 (1974).

[26] V.A. Miransky, "Dynamical Symmetry Breaking in Quantum Field Theories" (World Scientific, Singapore, 1993).

[27] "Dynamical Gauge Symmetry Breaking", edited by E. Farhi and R. Jackiw (World Scientific, Singapore, 1982).

[28] R. Jackiw and K. Johnson, Phys. Rev. D 8, 2386 (1973); J. Cornwall and R. Norton, Phys. Rev. D 8, 3338 (1973).

[29] G.'t Hooft, Nucl. Phys. B190 [FS3], 455 (1981).

[30] H. Bateman and A. Erdélyi, "Higher Transcendental Functions", v.1 (McGraw-Hill, New York, 1953).

[31] B. Simon, Ann. Phys. 97, 279 (1976).

[32] I.S. Gradshtein and I.M. Ryzhik, "Table of Integrals, Series and Products" (Academic Press, Orlando, 1980).

[33] G. Vafa and E. Witten, Nucl. Phys. B234, 173 (1984).

[34] T. Appelquist, M. Bowick, D. Karabali, and L.C.R. Wijewardhana, Phys. Rev. D 33, 3704 (1986). 

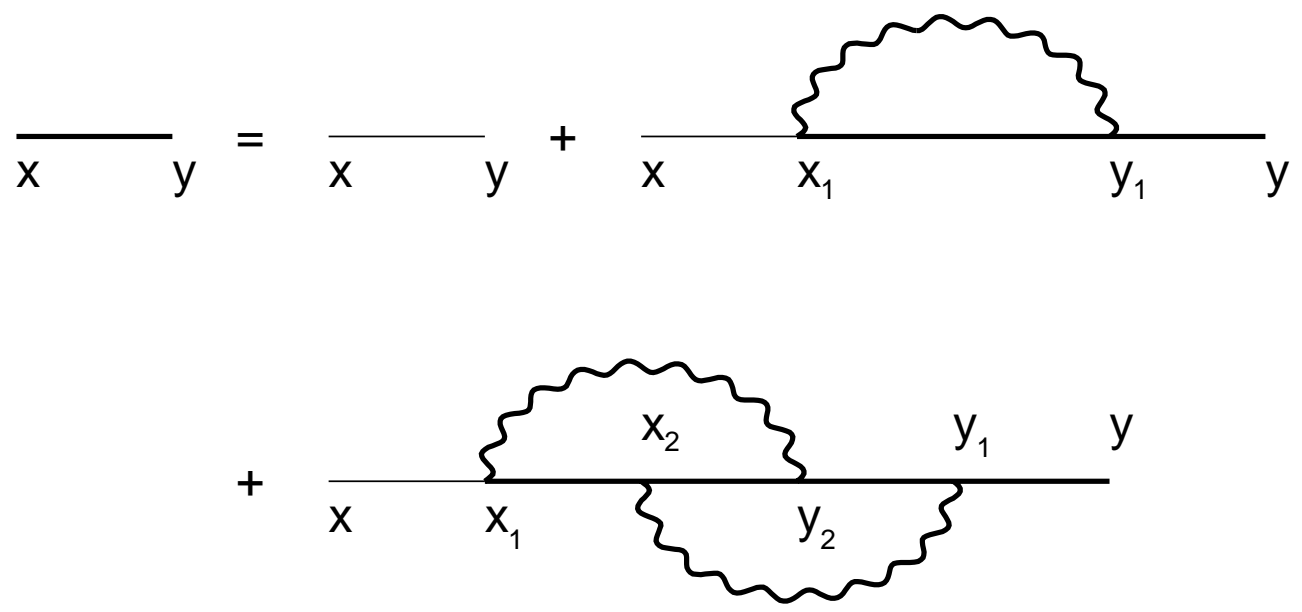

FIG. 1. The diagrammatic representation of the SD equation in coordinate space at two-loop order. A thin solid line corresponds to the bare fermion propagator; a bold line corresponds to the full fermion propagator, and a wavy line designs the full photon propagator.

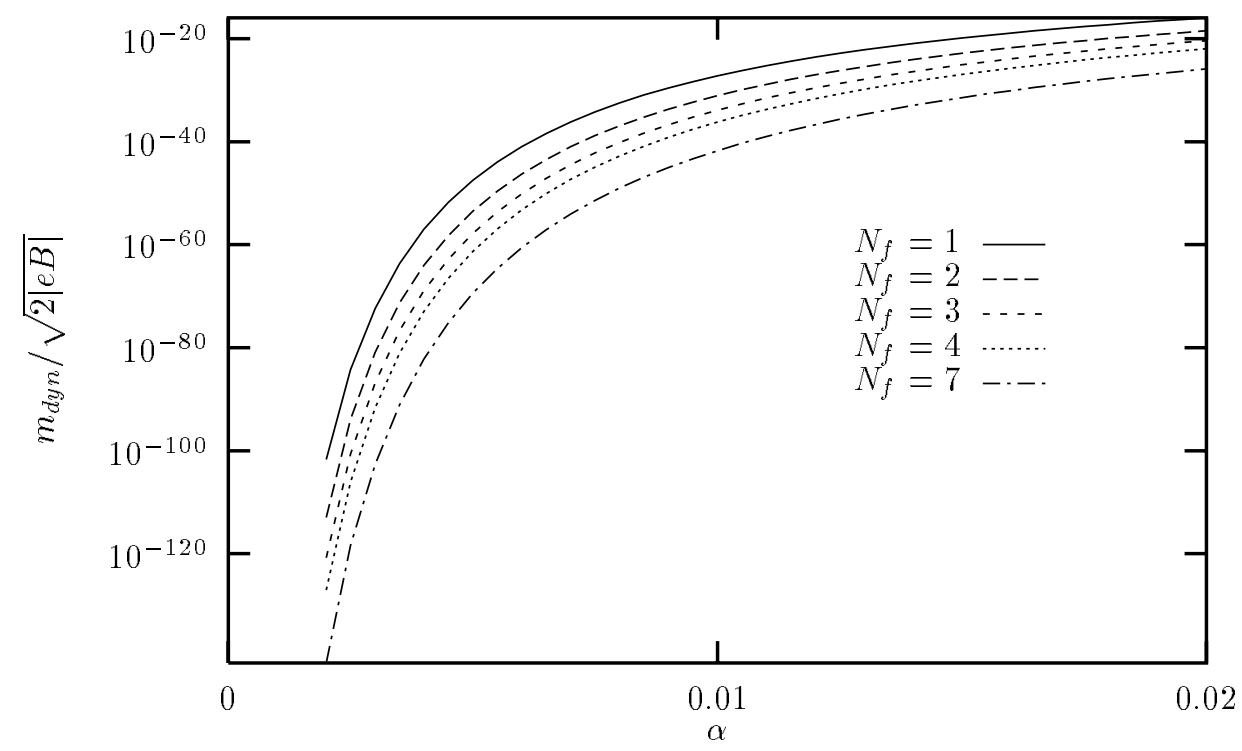

FIG. 2. Plot of $m_{d y n}$ as a function of $\alpha$ for several values of $N_{f}$. 


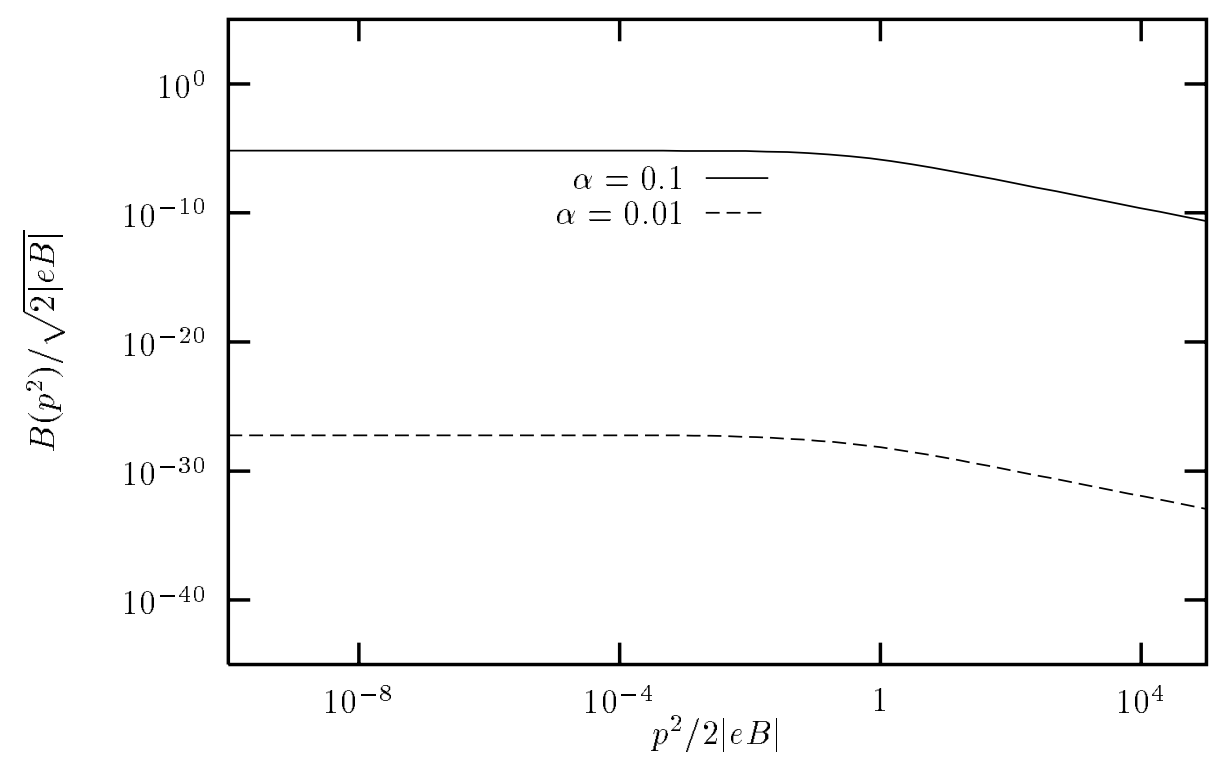

FIG. 3. Plot of the mass function $B\left(p^{2}\right)$ as a function of $p^{2}$ for $N_{f}=1$ and two values of $\alpha$.

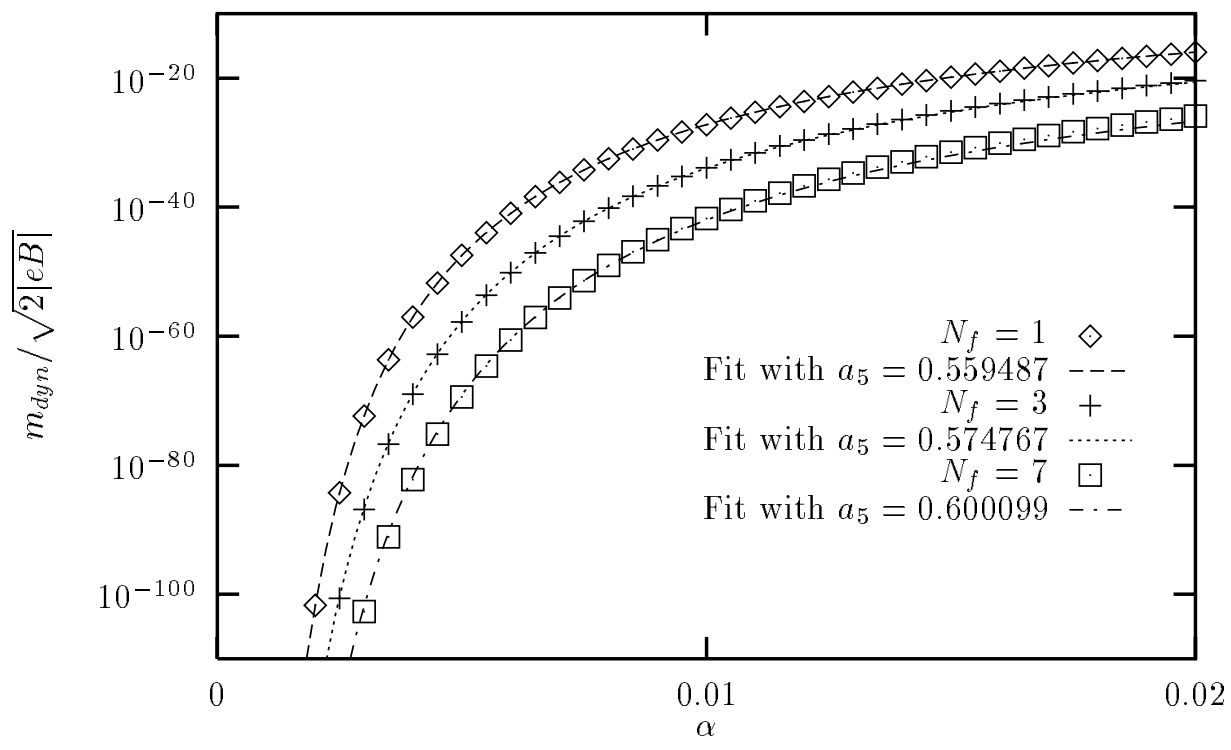

FIG. 4. Plot of the fit function and corresponding datapoints for several values of $N_{f}$ when the only free parameter was $a_{5}$. 


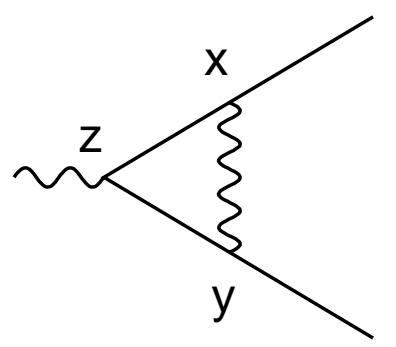

(a)

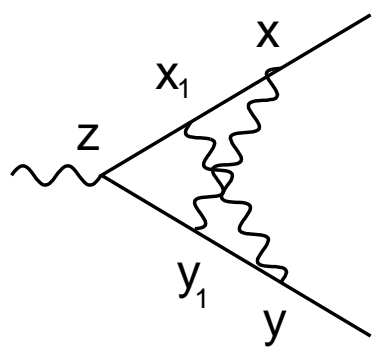

(b)

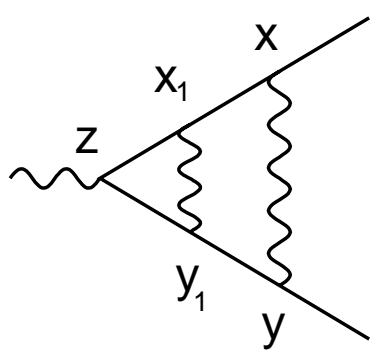

(c)

FIG. 5. The diagrammatic representation of the one- and two-loop corrections to the vertex. 